\title{
Effect of road transport for up to 24 hours followed by twenty-four hour recovery on live weight and physiological responses of bulls
}

\author{
Bernadette Earley*, Margaret Murray, Daniel J Prendiville
}

\begin{abstract}
Background: The transport of livestock can have major implications for their welfare, and there is strong public interest and scientific endeavour aimed at ensuring that the welfare of transported animals is optimal. The objective of the study was to investigate the effect of transport on live weight, physiological and haematological responses of bulls after road transport of $0,6,9,12,18$ and 24 hours (h). Seventy-two Charolais bulls (mean weight (s.d.) $367(35) \mathrm{kg}$ ), naïve to transport, were randomly assigned to one of six journey (J) times of $0 \mathrm{~h}, 6 \mathrm{~h}, 9 \mathrm{~h}, 12 \mathrm{~h}$, $18 \mathrm{~h}$ and $24 \mathrm{~h}$ transport ( $\mathrm{n}=12$ animals/treatment) at a stocking density of $1.02 \mathrm{~m}^{2} / \mathrm{bull}$. Blood samples were collected by jugular venipuncture before transport (-0.25 h), immediately after $(0 \mathrm{~h})$ and at $1 \mathrm{~h}, 2 \mathrm{~h}, 4 \mathrm{~h}, 6 \mathrm{~h}, 8 \mathrm{~h}$, $12 \mathrm{~h}$ and $24 \mathrm{~h}$ relative to time $0 \mathrm{~h}$. The bulls were weighed before transport ( $24 \mathrm{~h}$ and $-0.25 \mathrm{~h}$ ), immediately after $(0 \mathrm{~h})$, and at $4 \mathrm{~h}, 12 \mathrm{~h}$ and $24 \mathrm{~h}$ relative to time $0 \mathrm{~h}$. Control animals were blood sampled before assignment $(-0.25 \mathrm{~h})$ to novel pens, after $(24 \mathrm{~h})$, and at $1 \mathrm{~h}, 2 \mathrm{~h}, 4 \mathrm{~h}, 6 \mathrm{~h}, 8 \mathrm{~h}, 12 \mathrm{~h}$ and $24 \mathrm{~h}$ relative to the $24 \mathrm{~h}$ sampling time point.

Results: Bulls travelling for $6 \mathrm{~h}(280 \mathrm{~km}), 9 \mathrm{~h}(435 \mathrm{~km}), 12 \mathrm{~h}(582 \mathrm{~km}), 18 \mathrm{~h}(902 \mathrm{~km})$ and $24 \mathrm{~h}(1192 \mathrm{~km})$ lost 4.7, 4.5, $5.7(P<0.05), 6.6(P<0.05)$ and $7.5(P<0.05)$ percentage $(\%)$ live weight compared with baseline. Live weight re-gained to pre-transport levels during the $24 \mathrm{~h}$ recovery period. Lymphocyte percentages were lower $(P<0.05)$ and neutrophil percentages were greater $(P<0.05)$ in all animals. Blood protein, glucose and NEFA concentrations and creatine kinase activity were greater $(P<0.05)$ in the bulls following transport and returned to baseline within $24 \mathrm{~h}$.

Conclusions: Under the conditions of the present study, transport of bulls on journeys by road, ranging from $6 \mathrm{~h}$ $(280 \mathrm{~km})$ to $24 \mathrm{~h}(1192 \mathrm{~km})$ duration, affected live weight, haematological and physiological measurements of metabolism and inflammation. Our findings showed that live weight and some physiological and haematological responses of bulls returned to pre-transport levels within $24 \mathrm{~h}$ with animals having had access to feed and water.
\end{abstract}

\section{Background}

Transportation of livestock involves several potential stressors that result in increased cortisol [1-7], mobilisation of energy and protein metabolism [7], and a challenged immune system [8-12] resulting in increased disease susceptibility [13]. Studies have been carried out to determine the optimum stocking density, the maximum duration of transportation, the timing of rest stops and which components of the transport process are the most stressful to cattle [3,4,14-17]. Physical factors such

\footnotetext{
* Correspondence: bernadette.earley@teagasc.ie

Animal and Bioscience Research Department, Animal \& Grassland Research and Innovation Centre, Teagasc, Grange, Dunsany, Co. Meath, Ireland
}

as noise or vibrations; psychological/emotional factors, such as unfamiliar environment or social regrouping; and climatic factors, such as temperature and humidity, are also involved in the transport process. Steers transported (12-18 mo old) by road for 5,10 and $15 \mathrm{~h}$ lost 4.6, 6.5 and $7.0 \%$ of their live weight; and recovery to pre-transport live weight took 5 days $[18,19]$. There are limited scientific data on the physiological and haematological recovery of animals after long durations of transport and in particular the physiological recovery of animals during the $24 \mathrm{~h}$ period post-transport.

The objective of the study was to evaluate the effect of transport on live weight, physiological and haematological

\section{() Biomed Central}


responses of bulls after road transport of $0,6,9,12,18$ and $24 \mathrm{~h}$ and on their physiological recovery during the $24 \mathrm{~h}$ period post-transport.

\section{Results}

\section{Environment}

The mean temperature of the shed environment where the animals were housed was $15.6^{\circ} \mathrm{C}$ (minimum $6.0^{\circ} \mathrm{C}$ and maximum $19.3^{\circ} \mathrm{C}$ ). The environmental data recorded during each transport journey are reported in Table 1. The levels of $\mathrm{CO}_{2}$ tended to increase with the longer journeys and values were numerically greater during the $24 \mathrm{~h}$ journey compared with the other journeys. The levels of $\mathrm{H}_{2} \mathrm{~S}$ and $\mathrm{NH}_{3}$ remained low throughout the series of transport journeys. Vapour density and ambient temperature during the $9 \mathrm{~h}$ transport journey (J 9) was numerically greater compared with the other journey durations.

\section{Water intake}

Table 2 shows the consumption of water during and after transport. Most of the transported treatments consumed water during the transport journeys except for the J 12 treatment which did not consume water during transport. Post-transport all treatment groups had numerically greater water intake volumes in the $1-4 \mathrm{~h}$ period post-transport.

\section{Live weight}

Significant $(\mathrm{P}<0.05)$ live weight loss was observed for the $\mathrm{J} 18$ and $\mathrm{J} 24$ treatments and only at $0 \mathrm{~h}$ post-transport (Table 3). Bulls travelling for 6, 9, 12, 18 and $24 \mathrm{~h}$ lost 4.6, 4.5, 5.7, 6.3 and $7.5 \%$ live weight $(\mathrm{P}<0.05)$ compared with baseline while control animals lost $5.4 \%$.
During the $24 \mathrm{~h}$ recovery period live weight was regained to pre-transport levels in all treatments at $4 \mathrm{~h}$ (Table 3).

\section{Rectal temperature data}

There was no effect $(\mathrm{P}>0.05)$ of transport on rectal temperature (data not shown).

\section{Physiological, metabolic and haematological variables}

In control animals $(\mathrm{J} 0)$, there was no change $(\mathrm{P}>0.05)$ in albumin concentration (Table 4). There were transient increases in albumin concentrations in animals transported for $6 \mathrm{~h}(\mathrm{~J} 6)$ at $0 \mathrm{~h}$ post-transport $(\mathrm{P}<0.05)$ and in animals transported for $9 \mathrm{~h}(\mathrm{~J} 9)$ at $0 \mathrm{~h}$ and $1 \mathrm{~h}$ post-transport compared with pre-transport baselines. Animals transported for $12 \mathrm{~h}$ (J 12) had greater ( $\mathrm{P}<$ 0.05 ) albumin concentrations at $0 \mathrm{~h}, 1 \mathrm{~h}$ and $2 \mathrm{~h}$ posttransport compared with baseline. Following transportation of animals for $18 \mathrm{~h}$ (J 18) albumin concentrations were greater $(\mathrm{P}<0.05)$ at $0 \mathrm{~h}$ and $1 \mathrm{~h}$ post-transport, whereas the J 24 animals had greater concentrations (P $<0.05$ ) at $0 \mathrm{~h}, 1 \mathrm{~h}$ and $2 \mathrm{~h}$ post-transport.

Globulin concentrations were unchanged $(\mathrm{P}>0.05)$ (Table 4) in non-transported control animals $(\mathrm{J} 0)$, and in animals transported for 6 (J 6), 9 (J 9) and $18 \mathrm{~h}$ (J 18) compared with their pre-transport baselines. There were transient increases in globulin concentrations in the J 12 treatment at $0 \mathrm{~h}$ and $1 \mathrm{~h}$ post-transport, compared with baseline $(\mathrm{P}<0.05)$, whereas the $\mathrm{J} 24$ treatment had greater $(\mathrm{P}<0.05)$ globulin concentrations at $0 \mathrm{~h}, 1 \mathrm{~h}, 2$ $\mathrm{h}$ and $4 \mathrm{~h}$ post-transport compared with baseline.

In non-transported control animals ( 0 ), there was no change $(\mathrm{P}>0.05)$ in total protein concentration (Table 4). There were transient increases in protein concentrations

Table 1 Environmental conditions recorded during housing and during transport

\begin{tabular}{|c|c|c|c|c|c|c|c|c|}
\hline \multirow[t]{2}{*}{$\begin{array}{l}\text { Transport } \\
\text { journeys }\end{array}$} & \multirow[t]{2}{*}{$\begin{array}{l}\text { Ambient Temperature } \\
\left({ }^{\circ} \mathrm{C}\right)\end{array}$} & \multicolumn{7}{|c|}{$\begin{array}{l}\text { Environmental conditions recorded during housing of control }(\mathrm{J} 0) \text { and during transport of } \mathrm{J} 6 \text {, } \\
\mathrm{J} 9, \mathrm{~J} 12, \mathrm{~J} 18 \text { and } \mathrm{J} 24 \text { treatments }\end{array}$} \\
\hline & & $\begin{array}{l}\mathrm{CO}_{2} \\
(\mathrm{ppm})\end{array}$ & $\begin{array}{l}\text { Relative } \\
\text { humidity } \\
(\%)\end{array}$ & $\begin{array}{l}\text { Temperature } \\
\left({ }^{\circ} \mathrm{C}\right)\end{array}$ & $\begin{array}{l}\text { Wind } \\
\text { speed } \\
(\mathrm{m} / \mathrm{s})\end{array}$ & $\begin{array}{l}\text { Vapour } \\
\text { density } \\
\left(\mathrm{g} / \mathrm{m}^{3}\right)\end{array}$ & $\begin{array}{l}\mathrm{H}_{2} \mathrm{~S} \\
(\mathrm{ppm})\end{array}$ & $\begin{array}{l}\mathrm{NH}_{3} \\
(\mathrm{ppm})\end{array}$ \\
\hline $\mathrm{J} 0$ & $12.0(1.7-20.2)$ & $\begin{array}{l}471.3(417.0- \\
655.0)\end{array}$ & $69.8(40.9-88.6)$ & $15.6(6.0-19.0)$ & $\begin{array}{l}0.4(0.0- \\
0.10)\end{array}$ & $4.3(2.8-8.6)$ & $\begin{array}{l}0.00(0.00- \\
0.05)\end{array}$ & $\begin{array}{l}0.0(0.0- \\
0.0)\end{array}$ \\
\hline J 6 & $8.4(6.1-10.3)$ & $\begin{array}{l}453.8(356.0- \\
897.0)\end{array}$ & 89.5 (72.5-99.9) & $7.6(4.7-10.8)$ & $\begin{array}{l}0.8(0.1- \\
2.5)\end{array}$ & $5.9(4.3-10.8)$ & $\begin{array}{l}0.01(0.00- \\
0.10)\end{array}$ & $\begin{array}{l}1.3(0.0- \\
5.7)\end{array}$ \\
\hline J 9 & $15.3(11.3-17.7)$ & $\begin{array}{l}531.6(388.0- \\
922.0)\end{array}$ & $93.8(81.1-99.9)$ & $\begin{array}{l}14.4(10.4- \\
17.7)\end{array}$ & $\begin{array}{l}0.6(0.0- \\
2.3)\end{array}$ & $\begin{array}{l}13.4(10.4- \\
15.9)\end{array}$ & $\begin{array}{l}0.00(0.00- \\
0.00)\end{array}$ & $\begin{array}{l}0.4(0.0- \\
2.9)\end{array}$ \\
\hline $\mathrm{J} 12$ & $7.8(0.7-14.2)$ & $\begin{array}{l}668.8(447.0- \\
1903.0)\end{array}$ & $74.7(61.5-89.2)$ & $8.6(3.5-12.6)$ & $\begin{array}{l}0.7(0.0- \\
3.0)\end{array}$ & $4.3(1.2-8.7)$ & $\begin{array}{l}0.01(0.00- \\
0.20)\end{array}$ & $\begin{array}{l}0.9(0.0- \\
7.7)\end{array}$ \\
\hline J 18 & $7.1(3.5-9.9)$ & $\begin{array}{l}545.5 \text { (399.0- } \\
858.0)\end{array}$ & $78.1(65.0-91.0)$ & $8.3(4.7-10.5)$ & $\begin{array}{l}0.8(0.0- \\
3.3)\end{array}$ & $4.6(0.5-8.5)$ & $\begin{array}{l}0.04(0.00- \\
0.20)\end{array}$ & $\begin{array}{l}0.0(0.0- \\
0.0)\end{array}$ \\
\hline J 24 & $4.9(-3.1-18.1)$ & $\begin{array}{l}770.9(401.0- \\
2373.0)\end{array}$ & $86.2(64.3-99.9)$ & $6.4(-0.1-16.0)$ & $\begin{array}{l}0.6(0.0- \\
3.1)\end{array}$ & $4.2(-1.1-12.2)$ & $\begin{array}{l}0.00(0.00- \\
0.00)\end{array}$ & $\begin{array}{l}0.1(0.0- \\
2.9)\end{array}$ \\
\hline
\end{tabular}

The values are expressed as mean with minimum and maximum values.

Control = J 0 not transported (control); J 6, J 9, J 12, J 18 and J $24=$ animals transported for journey durations of $6,9,12,18$ and 24 hours (h) by road at a stocking density of $1.02 \mathrm{~m}^{2}$ per animal. 
Table 2 Water consumption (litres) per animal ( $n=12$ animals per treatment) during and after different transport journey $(J)$ durations

\begin{tabular}{|c|c|c|c|c|c|}
\hline \multirow[t]{2}{*}{$\begin{array}{l}\text { J duration } \\
\text { (hour (h)) }\end{array}$} & \multirow[t]{2}{*}{ Water intake (litres) during transport } & \multirow[t]{2}{*}{ Distance travelled by road $(\mathbf{k m})$} & \multicolumn{3}{|c|}{$\begin{array}{c}\text { Post-transport } \\
\text { (water intake (litres)) }\end{array}$} \\
\hline & & & $0-4 \mathrm{~h}$ & $5-12 h$ & $13-24 \mathrm{~h}$ \\
\hline $\mathrm{Oh}$ & 4.1 & 0 & $20.2^{a}$ & $4.5^{b}$ & $0.0^{b}$ \\
\hline $6 \mathrm{~h}$ & 5.0 & 280 & $8.0^{\mathrm{a}}$ & $3.5^{\mathrm{b}}$ & $0.5^{\mathrm{b}}$ \\
\hline $9 \mathrm{~h}$ & 5.0 & 435 & $11.5^{\mathrm{a}}$ & $1.5^{\mathrm{b}}$ & $6.0^{\mathrm{b}}$ \\
\hline $12 \mathrm{~h}$ & 0.0 & 582 & $8.5^{a}$ & $6.5^{\mathrm{ab}}$ & $4.0^{\mathrm{b}}$ \\
\hline $18 \mathrm{~h}$ & 1.8 & 902 & $5.5^{\mathrm{a}}$ & $7.0^{\mathrm{ab}}$ & $3.0^{\mathrm{b}}$ \\
\hline $24 \mathrm{~h}$ & 3.9 & 1192 & $9.0^{\mathrm{a}}$ & $7.0^{\mathrm{ab}}$ & $1.0^{c}$ \\
\hline
\end{tabular}

Control $=\mathrm{J} 0$ not transported (control); J 6, J 9, J 12, J 18 and $\mathrm{J} 24=$ animals transported for journey durations of $6,9,12,18$ and 24 hours by road at a stocking density of $1.02 \mathrm{~m}^{2}$ per animal.

$a, b$, within row means not having a common superscript differ significantly $(P<0.05)$.

for the $6 \mathrm{~h}(\mathrm{~J} 6)$ and $9 \mathrm{~h}(\mathrm{~J} 9)$ treatments at $0 \mathrm{~h}$ and $1 \mathrm{~h}$ post-transport compared with their pre-transport baselines $(\mathrm{P}<0.05)$. Animals transported for $12 \mathrm{~h}(\mathrm{~J} 12)$ had greater $(\mathrm{P}<0.05)$ total protein concentrations at $0 \mathrm{~h}, 1 \mathrm{~h}$ and $2 \mathrm{~h}$ post-transport compared with baseline. Following transportation of animals for $18 \mathrm{~h}$ (J 18) total protein concentrations were greater $(\mathrm{P}<0.05)$ at $0 \mathrm{~h}$ and $1 \mathrm{~h}$ posttransport compared with pre-transport values whereas protein concentrations were greater for the $24 \mathrm{~h}$ (J 24) treatment at $0 \mathrm{~h}, 1 \mathrm{~h}, 2 \mathrm{~h}, 4 \mathrm{~h}$ and $8 \mathrm{~h}(\mathrm{P}<0.05)$ posttransport compared with baseline.

In the non-transported controls (J 0), J 18 and J 24 treatments, urea concentrations were greater $(\mathrm{P}<0.05)$ than baseline at $0 \mathrm{~h}$ to $12 \mathrm{~h}$ and were not different $(\mathrm{P}>$ $0.05)$ at $24 \mathrm{~h}$ (Table 5). Animals transported for $6 \mathrm{~h}$ (J 6) had greater $(\mathrm{P}<0.05)$ urea concentrations at $0 \mathrm{~h}$ to $12 \mathrm{~h}$ post-transport while the $\mathrm{J} 9$ treatment had greater $(\mathrm{P}<0.05)$ urea concentrations at $0 \mathrm{~h}, 1 \mathrm{~h}$ and $2 \mathrm{~h}$ posttransport compared with pre-transport baselines. Animals transported for $12 \mathrm{~h}(\mathrm{~J} 12)$ had greater $(\mathrm{P}<0.05)$ urea concentrations at $0 \mathrm{~h}$ to $8 \mathrm{~h}$ post-transport compared with baseline.

$\beta \mathrm{HB}$ concentrations were greater $(\mathrm{P}<0.05)$ at $6 \mathrm{~h}$ to $24 \mathrm{~h}$ following the end of the $24 \mathrm{~h}$ experimental period in non-transported control animals ( $\mathrm{J}$ 0), when compared with baseline concentrations (Table 5). Animals transported for $6 \mathrm{~h} \mathrm{(J} \mathrm{6)} \mathrm{and} 9 \mathrm{~h} \mathrm{(J} \mathrm{9)} \mathrm{had} \mathrm{greater}$ $(\mathrm{P}<0.05) \beta \mathrm{HB}$ concentrations than baseline at $2 \mathrm{~h}$ to $24 \mathrm{~h}$ post-transport, whereas, animals transported for $12 \mathrm{~h}$ (J 12) had greater $(\mathrm{P}<0.05) \beta \mathrm{HB}$ concentrations than baseline at $4 \mathrm{~h}$ to $24 \mathrm{~h}$ post-transport. Following transportation of animals for $18 \mathrm{~h}(\mathrm{~J} 18) \beta \mathrm{HB}$ concentrations were greater $(\mathrm{P}<0.05)$ than baseline at $6 \mathrm{~h}$ to 24 $\mathrm{h}$ and animals transported for $24 \mathrm{~h}$ had greater $(\mathrm{P}<$ $0.05) \beta \mathrm{HB}$ concentrations than baseline at $8 \mathrm{~h}, 12 \mathrm{~h}$ and $24 \mathrm{~h}$ post-transport compared with baseline.

CK activities were greater $(\mathrm{P}<0.05)$ than baseline in non-transported control animals $(\mathrm{J} 0)$ at $0 \mathrm{~h}$ to $8 \mathrm{~h}$ post the $24 \mathrm{~h}$ experimental rest period (Table 5). Post-transport, $\mathrm{CK}$ activities were greater $(\mathrm{P}<0.05)$ than baseline for the J 6 treatment at $4 \mathrm{~h}$ to $12 \mathrm{~h}$, for the J 9 treatment at $1 \mathrm{~h}$ post-transport and for the J 12 treatment at $2 \mathrm{~h}$ to $8 \mathrm{~h}$ post-transport. Animals transported for $18 \mathrm{~h}$ (J 18) had greater $(\mathrm{P}<0.05)$ CK activities than baseline at $2 \mathrm{~h}$ to $12 \mathrm{~h}$ post-transport whereas levels were greater $(\mathrm{P}<0.05)$ for the $24 \mathrm{~h}$ treatment at $0 \mathrm{~h}$ to $12 \mathrm{~h}$ posttransport compared with baseline.

Haptoglobin concentrations were greater $(\mathrm{P}<0.05)$ than baseline in non-transported control animals at $24 \mathrm{~h}$ following the end of the experimental $24 \mathrm{~h}$ rest

Table 3 Mean live weight $(\mathrm{kg}) \pm$ s.d. in control and transported bulls $(\mathrm{n}=12$ animals per treatment) for the respective journey $(\mathrm{J})$ times pre- and post-transport

\begin{tabular}{|c|c|c|c|c|c|c|}
\hline \multicolumn{3}{|c|}{ Pre-transport } & \multicolumn{4}{|c|}{ Post-transport } \\
\hline $\begin{array}{l}\text { Journey } \\
(J)\end{array}$ & $-24 h$ & $-0.25 \mathrm{~h}$ & $\mathrm{Oh}$ & $4 \mathrm{~h}$ & $12 \mathrm{~h}$ & $24 \mathrm{~h}$ \\
\hline $\mathrm{JO}$ & $370.5 \pm 49.8$ & $373.2 \pm 48.5$ & $352.8 \pm 45.8$ & $360.1 \pm 48.4$ & $366.8 \pm 48.9$ & $356.9 \pm 46.5$ \\
\hline 16 & $370.8 \pm 32.6$ & $367.1 \pm 31.8$ & $349.8 \pm 29.6$ & $362.2 \pm 30.1$ & $363.2 \pm 32.6$ & $362.2 \pm 33.4$ \\
\hline J 9 & $369.3 \pm 50.1$ & $362.4 \pm 50.9$ & $346.2 \pm 48.2$ & $359.2 \pm 48.0$ & $355.2 \pm 48.9$ & $361.2 \pm 50.0$ \\
\hline $\mathrm{J} 12$ & $367.7 \pm 26.2$ & $367.0 \pm 26.6$ & $346.1 \pm 26.1$ & $360.8 \pm 27.1$ & $359.7 \pm 27.1$ & $365.8 \pm 27.5$ \\
\hline J 18 & $365.2 \pm 27.0^{a}$ & $363.8 \pm 26.3^{a}$ & $339.5 \pm 22.7^{b}$ & $345.6 \pm 24.0^{\mathrm{ab}}$ & $350.1 \pm 24.9^{\mathrm{ab}}$ & $349.3 \pm 25.8^{\mathrm{ab}}$ \\
\hline J 24 & $362.8 \pm 21.1^{a}$ & $359.7 \pm 20.7^{a}$ & $332.6 \pm 19.6^{b}$ & $346.4 \pm 19.5^{\mathrm{ab}}$ & $354.7 \pm 22.2^{\mathrm{ab}}$ & $352.7 \pm 21.2^{\mathrm{ab}}$ \\
\hline
\end{tabular}

Control = J 0 not transported; animals transported for journey $(\mathrm{J})$ durations of $6,9,12,18$ and 24 hours (h) by road at a stocking density of $1.02 \mathrm{~m}^{2}$ per animal. ${ }^{a}$

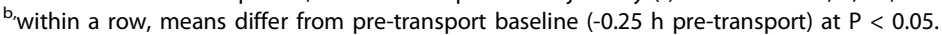


Table 4 Treatment means ( $n=12$ animals per treatment) with $\mathrm{SE}^{1}$ for plasma albumin, globulin and total protein pre $(-0.25 \mathrm{~h})$ and post-transport $(0,1,2,4,6,8,12$ and $24 \mathrm{~h})$

\begin{tabular}{|c|c|c|c|c|c|c|c|c|c|c|c|}
\hline Transport & $-0.25 \mathrm{~h}$ pre & $0 \mathrm{~h}$ post & $1 \mathrm{~h}$ post & $2 \mathrm{~h}$ post & $4 \mathrm{~h}$ post & $6 \mathrm{~h}$ post & $8 \mathrm{~h}$ post & $12 \mathrm{~h}$ post & $24 \mathrm{~h}$ post & $\mathrm{SE}^{1}$ & Effect of time ${ }^{2}$ \\
\hline \multicolumn{12}{|c|}{ Albumin $(\mathrm{g} / \mathrm{L})$} \\
\hline J 0 & 26.4 & 27.1 & 27.3 & 26.8 & 27.3 & 27.3 & 27.1 & 27.6 & 26.9 & 0.57 & $P=0.95$ \\
\hline 16 & $27.7^{\mathrm{a}}$ & $29.6^{\mathrm{b}}$ & $29.4^{\mathrm{ab}}$ & $29.2^{\mathrm{ab}}$ & $29.1^{\mathrm{ab}}$ & $28.5^{\mathrm{ab}}$ & $27.6^{\mathrm{ab}}$ & $27.8^{\mathrm{ab}}$ & $27.5^{\mathrm{ab}}$ & 0.64 & $P=0.10$ \\
\hline J 9 & $27.1^{\mathrm{a}}$ & $29.3^{b}$ & $29.11^{b}$ & $28.4^{\mathrm{ab}}$ & $27.7^{\mathrm{ab}}$ & $27.6^{\mathrm{ab}}$ & $27.7^{\mathrm{ab}}$ & $27.5^{\mathrm{ab}}$ & $27.6^{\mathrm{ab}}$ & 0.68 & $P=0.28$ \\
\hline J 12 & $27.8^{\mathrm{a}}$ & $29.8^{\mathrm{b}}$ & $30.0^{\mathrm{b}}$ & $29.5^{\mathrm{b}}$ & $28.8^{\mathrm{ab}}$ & $28.3^{\mathrm{ab}}$ & $28.6^{\mathrm{ab}}$ & $28.3^{\mathrm{ab}}$ & $28.5^{\mathrm{ab}}$ & 0.43 & $P=0.01$ \\
\hline J 18 & $27.3^{\mathrm{a}}$ & $29.7^{b}$ & $29.3^{b}$ & $28.7^{\mathrm{ab}}$ & $28.9^{\mathrm{ab}}$ & $28.7^{\mathrm{ab}}$ & $28.5^{\mathrm{ab}}$ & $28.3^{\mathrm{ab}}$ & $27.4^{\mathrm{ab}}$ & 0.58 & $P=0.08$ \\
\hline J 24 & $27.8^{\mathrm{b}}$ & $29.7^{b}$ & $29.9^{b}$ & $30.2^{b}$ & $28.9^{\mathrm{ab}}$ & $28.8^{\mathrm{ab}}$ & $28.9^{\mathrm{ab}}$ & $28.4^{\mathrm{ab}}$ & $28.3^{\mathrm{ab}}$ & 0.61 & $P=0.10$ \\
\hline \multicolumn{12}{|c|}{ Globulin ( $\mathrm{g} / \mathrm{L})$} \\
\hline J 0 & 40.0 & 41.4 & 40.2 & 40.6 & 40.6 & 41.0 & 40.0 & 41.6 & 41.3 & 0.86 & $P=0.85$ \\
\hline 16 & 39.2 & 41.1 & 41.0 & 39.9 & 39.0 & 38.6 & 38.8 & 38.4 & 37.8 & 0.93 & $P=0.18$ \\
\hline J 9 & 40.3 & 43.2 & 42.6 & 41.9 & 40.3 & 39.7 & 40.1 & 40.7 & 41.0 & 1.43 & $P=0.68$ \\
\hline J 12 & $37.6^{\mathrm{a}}$ & $41.3^{b}$ & $41.0^{\mathrm{b}}$ & $40.1^{\mathrm{ab}}$ & $39.0^{\mathrm{ab}}$ & $38.7^{\mathrm{ab}}$ & $38.9^{\mathrm{ab}}$ & $38.9^{\mathrm{ab}}$ & $39.4^{\mathrm{ab}}$ & 0.98 & $P=0.19$ \\
\hline J 18 & 39.9 & 43.4 & 42.6 & 42.6 & 42.2 & 41.5 & 41.6 & 41.8 & 40.7 & 1.79 & $P=0.95$ \\
\hline J 24 & $39.3^{\mathrm{a}}$ & $43.1^{\mathrm{b}}$ & $43.1^{\mathrm{b}}$ & $43.2^{\mathrm{b}}$ & $41.7^{\mathrm{b}}$ & $40.5^{\mathrm{ab}}$ & $41.1^{\mathrm{ab}}$ & $40.9^{\mathrm{ab}}$ & $40.7^{\mathrm{ab}}$ & 0.81 & $P=0.01$ \\
\hline \multicolumn{12}{|c|}{ Total protein $(\mathrm{g} / \mathrm{L})$} \\
\hline J 0 & 66.4 & 68.5 & 67.5 & 67.5 & 67.9 & 68.4 & 67.1 & 69.1 & 68.2 & 1.07 & $P=0.79$ \\
\hline 16 & $66.9^{\mathrm{a}}$ & $70.6^{\mathrm{b}}$ & $70.4^{\mathrm{b}}$ & $69.2^{\mathrm{ab}}$ & $68.3^{\mathrm{ab}}$ & $67.1^{\mathrm{ab}}$ & $66.4^{\mathrm{ab}}$ & $66.2^{\mathrm{ab}}$ & $65.3^{\mathrm{ab}}$ & 0.88 & $P=0.0001$ \\
\hline J 9 & $67.4^{\mathrm{a}}$ & $72.5^{\mathrm{b}}$ & $71.7^{\mathrm{b}}$ & $70.3^{\mathrm{ab}}$ & $67.9^{\mathrm{ab}}$ & $67.3^{\mathrm{ab}}$ & $67.8^{\mathrm{ab}}$ & $68.2^{\mathrm{ab}}$ & $68.6^{\mathrm{ab}}$ & 1.18 & $P=0.01$ \\
\hline J 12 & $65.4^{\mathrm{a}}$ & $71.1^{\mathrm{b}}$ & $70.9^{b}$ & $69.5^{\mathrm{b}}$ & $67.7^{\mathrm{ab}}$ & $67.0^{\mathrm{ab}}$ & $67.4^{\mathrm{ab}}$ & $67.2^{\mathrm{ab}}$ & $67.8^{\mathrm{ab}}$ & 1.00 & $P=0.001$ \\
\hline 18 & $67.14^{\mathrm{a}}$ & $73.0^{\mathrm{b}}$ & $71.9^{\mathrm{b}}$ & $71.4^{\mathrm{ab}}$ & $71.0^{\mathrm{ab}}$ & $70.2^{\mathrm{ab}}$ & $70.1^{\mathrm{ab}}$ & $70.1^{\mathrm{ab}}$ & $68.2^{\mathrm{ab}}$ & 1.61 & $P=0.27$ \\
\hline J 24 & $67.1^{\mathrm{a}}$ & $72.8^{\mathrm{b}}$ & $73.0^{\mathrm{b}}$ & $73.4^{\mathrm{b}}$ & $70.6^{b}$ & $69.3^{\mathrm{ab}}$ & $70.0^{\mathrm{b}}$ & $69.3^{\mathrm{ab}}$ & $69.0^{\mathrm{ab}}$ & 0.93 & $P=0.0001$ \\
\hline
\end{tabular}

Control = J 0 not transported; animals transported for journey (J) durations of $6,9,12,18$ and 24 hours (h) by road at a stocking density of 1.02 m ${ }^{2}$ per animal.

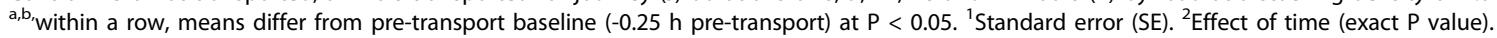

Table 5 Treatment means ( $\mathrm{n}=12$ animals per treatment) with $\mathrm{SE}^{1}$ for plasma urea, $\beta \mathrm{HB}$ and creatine kinase activity pre $(-0.25 \mathrm{~h})$ and post-transport $(0,1,2,4,6,8,12$ and $24 \mathrm{~h})$

\begin{tabular}{|c|c|c|c|c|c|c|c|c|c|c|c|}
\hline Transport & $-0.25 \mathrm{~h}$ pre & $0 \mathrm{~h}$ post & $1 \mathrm{~h}$ post & $2 \mathrm{~h}$ post & $4 \mathrm{~h}$ post & $6 \mathrm{~h}$ post & $8 \mathrm{~h}$ post & $12 \mathrm{~h}$ post & $24 \mathrm{~h}$ post & $\mathrm{SE}^{1}$ & Effect of time ${ }^{2}$ \\
\hline \multicolumn{12}{|c|}{ Urea (mmol/L) } \\
\hline $\mathrm{J} 0$ & $2.3^{\mathrm{a}}$ & $4.1^{\mathrm{b}}$ & $4.1^{\mathrm{b}}$ & $4.3^{b}$ & $4.6^{\mathrm{b}}$ & $4.6^{\mathrm{b}}$ & $4.3^{b}$ & $3.5^{\mathrm{b}}$ & $2.9^{\mathrm{ab}}$ & 0.29 & $P=0.001$ \\
\hline 16 & $2.4^{\mathrm{a}}$ & $2.9^{\mathrm{b}}$ & $3.0^{\mathrm{b}}$ & $3.2^{b}$ & $3.7^{\mathrm{b}}$ & $4.2^{\mathrm{b}}$ & $4.5^{\mathrm{b}}$ & $4.4^{\mathrm{b}}$ & $3.6^{\mathrm{ab}}$ & 0.14 & $P=0.001$ \\
\hline J 9 & $3.2^{\mathrm{a}}$ & $3.5^{\mathrm{b}}$ & $3.6^{b}$ & $3.9^{\mathrm{b}}$ & $4.1^{\mathrm{ab}}$ & $4.4^{\mathrm{ab}}$ & $4.3^{\mathrm{ab}}$ & $3.7^{\mathrm{ab}}$ & $2.9^{\mathrm{ab}}$ & 0.25 & $P=0.001$ \\
\hline J 12 & $3.3^{\mathrm{a}}$ & $4.1^{\mathrm{b}}$ & $4.3^{b}$ & $4.5^{b}$ & $4.8^{\mathrm{b}}$ & $4.7^{\mathrm{b}}$ & $4.2^{\mathrm{b}}$ & $3.5^{\mathrm{ab}}$ & $2.6^{\mathrm{ab}}$ & 0.19 & $P=0.001$ \\
\hline J 18 & $2.2^{a}$ & $4.3^{b}$ & $4.5^{\mathrm{b}}$ & $4.6^{\mathrm{b}}$ & $4.8^{\mathrm{b}}$ & $4.5^{\mathrm{b}}$ & $4.0^{b}$ & $3.2^{b}$ & $2.3^{\mathrm{ab}}$ & 0.17 & $P=0.001$ \\
\hline $\mathrm{J} 24$ & $2.4^{\mathrm{a}}$ & $5.4^{\mathrm{b}}$ & $5.4^{b}$ & $5.5^{\mathrm{b}}$ & $5.7^{\mathrm{b}}$ & $5.7^{\mathrm{b}}$ & $5.4^{\mathrm{b}}$ & $4.3^{b}$ & $2.8^{\mathrm{ab}}$ & 0.27 & $P=0.001$ \\
\hline \multicolumn{12}{|c|}{$\beta \mathrm{HB}(\mathrm{mmol} / \mathrm{L})$} \\
\hline J 0 & $0.18^{\mathrm{a}}$ & $0.22^{\mathrm{ab}}$ & $0.20^{\mathrm{ab}}$ & $0.19^{\mathrm{ab}}$ & $0.25^{\mathrm{ab}}$ & $0.29^{b}$ & $0.36^{b}$ & $0.28^{\mathrm{b}}$ & $0.29^{b}$ & 0.03 & $P=0.001$ \\
\hline 16 & $0.17^{\mathrm{a}}$ & $0.16^{\mathrm{ab}}$ & $0.17^{\mathrm{ab}}$ & $0.22^{\mathrm{b}}$ & $0.25^{b}$ & 0.25 & $0.25^{\mathrm{b}}$ & $0.24^{b}$ & $0.26^{b}$ & 0.01 & $P=0.001$ \\
\hline J 9 & $0.18^{\mathrm{a}}$ & $0.16^{\mathrm{ab}}$ & $0.19^{\mathrm{ab}}$ & $0.25^{\mathrm{b}}$ & $0.31^{b}$ & $0.32^{\mathrm{b}}$ & $0.30^{\mathrm{b}}$ & $0.28^{\mathrm{b}}$ & $0.30^{\mathrm{b}}$ & 0.02 & $P=0.001$ \\
\hline $\mathrm{J} 12$ & $0.20^{\mathrm{a}}$ & $0.16^{\mathrm{ab}}$ & $0.17^{\mathrm{ab}}$ & $0.23^{\mathrm{ab}}$ & $0.28^{\mathrm{b}}$ & $0.30^{\mathrm{b}}$ & $0.29^{b}$ & $0.25^{b}$ & $0.32^{b}$ & 0.15 & $P=0.001$ \\
\hline $\mathrm{J} 18$ & $0.18^{\mathrm{b}}$ & $0.20^{\mathrm{ab}}$ & $0.14^{\mathrm{ab}}$ & $0.18^{\mathrm{ab}}$ & $0.20^{\mathrm{ab}}$ & $0.28^{b}$ & $0.27^{b}$ & $0.28^{\mathrm{b}}$ & $0.31^{b}$ & 0.02 & $P=0.001$ \\
\hline J 24 & $0.20^{\mathrm{a}}$ & $0.18^{\mathrm{ab}}$ & $0.14^{\mathrm{ab}}$ & $0.17^{\mathrm{ab}}$ & $0.22^{\mathrm{ab}}$ & $0.27^{\mathrm{ab}}$ & $0.36^{b}$ & $0.39^{b}$ & $0.34^{\mathrm{b}}$ & 0.20 & $P=0.001$ \\
\hline \multicolumn{12}{|l|}{ CK (U/L) } \\
\hline J 0 & $159.6^{\mathrm{a}}$ & $437.1^{b}$ & $511.4^{\mathrm{b}}$ & $551.4^{\mathrm{b}}$ & $552.4^{b}$ & $524.8^{b}$ & $483.6^{\mathrm{b}}$ & $430.4^{\mathrm{ab}}$ & $381.0^{\mathrm{ab}}$ & 60.3 & $P=0.86$ \\
\hline 16 & $160.6^{\mathrm{a}}$ & $377.9^{\mathrm{ab}}$ & $443.9^{\mathrm{ab}}$ & $521.1^{\mathrm{ab}}$ & $627.0^{b}$ & $691.4^{b}$ & $605.6^{b}$ & $487.42^{\mathrm{b}}$ & $427.2^{\mathrm{ab}}$ & 69.5 & $P=0.44$ \\
\hline J 9 & $242.2^{\mathrm{a}}$ & $428.3^{\mathrm{ab}}$ & $522.7^{b}$ & $469.7^{\mathrm{ab}}$ & $511.1^{\mathrm{ab}}$ & $469.0^{\mathrm{ab}}$ & $357.4^{\mathrm{ab}}$ & $364.5^{\mathrm{ab}}$ & $319.6^{\mathrm{ab}}$ & 286.0 & $P=0.53$ \\
\hline $\mathrm{J} 12$ & $187.7^{\mathrm{a}}$ & $368.5^{\mathrm{ab}}$ & $398.2^{\mathrm{ab}}$ & $548.3^{b}$ & $545.0^{\mathrm{b}}$ & $497.3^{b}$ & $494.8^{b}$ & $431.7^{\mathrm{b}}$ & $404.6^{\mathrm{ab}}$ & 77.5 & $P=0.04$ \\
\hline J 18 & $159.6^{a}$ & $472.9^{\mathrm{ab}}$ & $497.1^{\mathrm{ab}}$ & $467.1^{b}$ & $445.3^{b}$ & $477.9^{b}$ & $475.7^{b}$ & $437.5^{b}$ & $393.4^{\mathrm{ab}}$ & 124.0 & $P=0.17$ \\
\hline J 24 & $162.3^{\mathrm{a}}$ & $496.20^{b}$ & $545.7^{b}$ & $598.6^{\mathrm{b}}$ & $598.7^{b}$ & $600.0^{\mathrm{b}}$ & $631.6^{\mathrm{b}}$ & $552.0^{\mathrm{b}}$ & $340.3^{\mathrm{ab}}$ & 92.8 & $P=0.02$ \\
\hline
\end{tabular}

Control = $\mathrm{J} 0$ not transported; animals transported for journey $(\mathrm{J})$ durations of $6,9,12,18$ and 24 hours (h) by road at a stocking density of $1.02 \mathrm{~m}^{2}$ per animal. ${ }^{a}, b_{\text {, }}$ within a row, means differ from pre-transport baseline $\left(-0.25 \mathrm{~h}\right.$ pre-transport) at $\mathrm{P}<0.05$. ${ }^{1}$ Standard error (SE). ${ }^{2}$ Effect of time (exact $\mathrm{P}$ value). 
Table 6 Treatment means ( $\mathrm{n}=12$ animals per treatment) with $\mathrm{SE}^{1}$ for plasma haptoglobin, glucose and NEFA pre $(-0.25 \mathrm{~h})$ and post-transport $(0,1,2,4,6,8,12$ and $24 \mathrm{~h})$

\begin{tabular}{|c|c|c|c|c|c|c|c|c|c|c|c|}
\hline Transport & $-0.25 \mathrm{~h}$ pre & $0 \mathrm{~h}$ post & $1 \mathrm{~h}$ post & $2 \mathrm{~h}$ post & $4 \mathrm{~h}$ post & $6 \mathrm{~h}$ post & $8 \mathrm{~h}$ post & $12 \mathrm{~h}$ post & $24 \mathrm{~h}$ post & $\mathrm{SE}^{1}$ & Effect of time ${ }^{2}$ \\
\hline \multicolumn{12}{|c|}{ Haptoglobin (Hgb binding capacity/L) } \\
\hline J 0 & $0.16^{\mathrm{a}}$ & $0.34^{\mathrm{ab}}$ & $0.34^{\mathrm{ab}}$ & $0.32^{\mathrm{ab}}$ & $0.34^{\mathrm{ab}}$ & $0.32^{\mathrm{ab}}$ & $0.33^{\mathrm{ab}}$ & $0.36^{\mathrm{ab}}$ & $0.60^{\mathrm{b}}$ & 0.09 & $P=0.19$ \\
\hline J 6 & 0.10 & 0.08 & 0.09 & 0.10 & 0.12 & 0.11 & 0.09 & 0.11 & 0.16 & 0.01 & $P=0.0002$ \\
\hline J 9 & 0.15 & 0.16 & 0.16 & 0.15 & 0.15 & 0.18 & 0.19 & 0.22 & 0.29 & 0.08 & $P=0.96$ \\
\hline J 12 & $0.10^{\mathrm{a}}$ & $0.14^{\mathrm{ab}}$ & $0.17^{\mathrm{b}}$ & $0.14^{\mathrm{ab}}$ & $0.13^{\mathrm{ab}}$ & $0.13^{\mathrm{ab}}$ & $0.14^{\mathrm{ab}}$ & $0.17^{b}$ & $0.23^{b}$ & 0.02 & $P=0.013$ \\
\hline J 18 & $0.09^{\mathrm{a}}$ & $0.16^{\mathrm{ab}}$ & $0.15^{\mathrm{ab}}$ & $0.13^{\mathrm{ab}}$ & $0.14^{\mathrm{ab}}$ & $0.14^{\mathrm{ab}}$ & $0.14^{\mathrm{ab}}$ & $0.14^{\mathrm{ab}}$ & $0.26^{\mathrm{b}}$ & 0.03 & $P=0.03$ \\
\hline J 24 & $0.05^{\mathrm{a}}$ & $0.12^{\mathrm{ab}}$ & $0.13^{\mathrm{ab}}$ & $0.12^{\mathrm{ab}}$ & $0.11^{\mathrm{ab}}$ & $0.14^{b}$ & $0.24^{b}$ & $0.25^{b}$ & $0.41^{\mathrm{b}}$ & 0.03 & $P=0.0001$ \\
\hline \multicolumn{12}{|c|}{ Glucose (mmol/L) } \\
\hline J 0 & $4.7^{\mathrm{a}}$ & $4.1^{\mathrm{b}}$ & $4.3^{\mathrm{ab}}$ & $4.3^{\mathrm{ab}}$ & $3.8^{\mathrm{b}}$ & $4.0^{b}$ & $4.0^{\mathrm{b}}$ & $4.1^{\mathrm{b}}$ & $4.4^{\mathrm{ab}}$ & 0.14 & $P=0.002$ \\
\hline 16 & $4.7^{\mathrm{a}}$ & $4.9^{\mathrm{ab}}$ & $5.4^{\mathrm{b}}$ & $5.4^{\mathrm{b}}$ & $4.9^{\mathrm{ab}}$ & $4.9^{\mathrm{ab}}$ & $4.9^{\mathrm{ab}}$ & $4.9^{\mathrm{ab}}$ & $4.2^{\mathrm{ab}}$ & 0.22 & $P=0.01$ \\
\hline J 9 & $4.4^{\mathrm{a}}$ & $5.7^{\mathrm{b}}$ & $5.5^{\mathrm{b}}$ & $5.2^{\mathrm{b}}$ & $4.7^{\mathrm{ab}}$ & $4.6^{\mathrm{ab}}$ & $4.4^{\mathrm{ab}}$ & $4.3^{\mathrm{ab}}$ & $4.3^{\mathrm{ab}}$ & 0.15 & $P=0.0001$ \\
\hline J 12 & $4.8^{\mathrm{a}}$ & $6.3^{\mathrm{b}}$ & $6.0^{\mathrm{b}}$ & $5.5^{\mathrm{b}}$ & $5.3^{\mathrm{ab}}$ & $4.9^{\mathrm{ab}}$ & $4.8^{\mathrm{ab}}$ & $4.6^{\mathrm{ab}}$ & $4.7^{\mathrm{ab}}$ & 0.20 & $P=0.0001$ \\
\hline J 18 & $4.7^{\mathrm{a}}$ & $5.7^{\mathrm{b}}$ & $5.9^{\mathrm{b}}$ & $5.1^{\mathrm{ab}}$ & $4.7^{\mathrm{ab}}$ & $4.5^{\mathrm{ab}}$ & $4.5^{\mathrm{ab}}$ & $4.2^{\mathrm{ab}}$ & $4.4^{\mathrm{ab}}$ & 0.22 & $P=0.0001$ \\
\hline J 24 & $4.3^{\mathrm{a}}$ & $5.8^{\mathrm{b}}$ & $5.4^{\mathrm{b}}$ & $5.1^{\mathrm{b}}$ & $4.8^{\mathrm{ab}}$ & $4.6^{\mathrm{ab}}$ & $4.2^{\mathrm{ab}}$ & $4.1^{\mathrm{ab}}$ & $4.2^{\mathrm{ab}}$ & 0.19 & $P=0.0001$ \\
\hline \multicolumn{12}{|c|}{ NEFA ( $\mu \mathrm{mol} / \mathrm{L})$} \\
\hline J 0 & $0.25^{\mathrm{a}}$ & $0.88^{\mathrm{b}}$ & $0.69^{b}$ & $0.42^{\mathrm{b}}$ & $0.34^{\mathrm{ab}}$ & $0.32^{\mathrm{ab}}$ & $0.29^{\mathrm{ab}}$ & $0.22^{\mathrm{ab}}$ & $0.56^{\mathrm{b}}$ & 0.05 & $P=0.0001$ \\
\hline 16 & $0.26^{\mathrm{a}}$ & $0.35^{\mathrm{ab}}$ & $0.19^{\mathrm{ab}}$ & $0.14^{\mathrm{ab}}$ & $0.15^{\mathrm{ab}}$ & $0.18^{\mathrm{ab}}$ & $0.25^{\mathrm{ab}}$ & $0.42^{\mathrm{b}}$ & $0.23^{\mathrm{ab}}$ & 0.04 & $P=0.0001$ \\
\hline J 9 & $0.24^{\mathrm{a}}$ & $0.32^{\mathrm{ab}}$ & $0.12^{\mathrm{b}}$ & $0.09^{b}$ & $0.10^{b}$ & $0.13^{b}$ & $0.15^{\mathrm{ab}}$ & $0.22^{\mathrm{ab}}$ & $0.22^{\mathrm{ab}}$ & 0.03 & $P=0.0001$ \\
\hline J 12 & $0.28^{a}$ & $0.32^{\mathrm{ab}}$ & $0.12^{\mathrm{b}}$ & $0.11^{\mathrm{b}}$ & $0.12^{\mathrm{b}}$ & $0.15^{b}$ & $0.21^{\mathrm{ab}}$ & $0.21^{\mathrm{ab}}$ & $0.35^{\mathrm{ab}}$ & 0.03 & $P=0.0001$ \\
\hline J 18 & $0.19^{a}$ & $0.40^{\mathrm{b}}$ & $0.17^{\mathrm{ab}}$ & $0.16^{\mathrm{ab}}$ & $0.23^{\mathrm{ab}}$ & $0.30^{\mathrm{ab}}$ & $0.25^{\mathrm{ab}}$ & $0.30^{\mathrm{ab}}$ & $0.55^{\mathrm{b}}$ & 0.06 & $P=0.0001$ \\
\hline J 24 & $0.27^{\mathrm{a}}$ & $0.44^{b}$ & $0.40^{\mathrm{b}}$ & $0.25^{\mathrm{ab}}$ & $0.22^{\mathrm{ab}}$ & $0.22^{\mathrm{ab}}$ & $0.15^{\mathrm{ab}}$ & $0.13^{\mathrm{ab}}$ & $0.48^{\mathrm{b}}$ & 0.04 & $P=0.0001$ \\
\hline
\end{tabular}

Control = J 0 not transported; animals transported for journey $(\mathrm{J})$ durations of $6,9,12,18$ and 24 hours (h) by road at a stocking density of 1.02 m ${ }^{2}$ per animal. ${ }^{a}, b_{\text {, }}$ within a row, means differ from pre-transport baseline $\left(-0.25 \mathrm{~h}\right.$ pre-transport) at $\mathrm{P}<0.05$. ${ }^{1}$ Standard error (SE). ${ }^{2}$ Effect of time (exact $\mathrm{P}$ value).

period (Table 6), and were unchanged in animals transported for 6 (J 6) and $9 \mathrm{~h}$ (J 9). There were transient increases $(\mathrm{P}<0.05)$ in haptoglobin concentrations for the J 12 treatment at $1 \mathrm{~h}, 12$ and $24 \mathrm{~h}$ post-transport, for the J 18 treatment at $24 \mathrm{~h}$ post-transport and for the J 24 treatment at 6 to $24 \mathrm{~h}$ post-transport compared with baseline.

In non-transported control animals (J 0), glucose concentrations were lower $(\mathrm{P}<0.05)$ than baseline at $0 \mathrm{~h}$, $4 \mathrm{~h}, 6 \mathrm{~h}, 8 \mathrm{~h}$ and $12 \mathrm{~h}$ relative to the end of the initial $24 \mathrm{~h}$ experimental period (Table 6 ). There were transient increases in glucose concentrations for the J 6 treatment at $1 \mathrm{~h}$ and $2 \mathrm{~h}$ post-transport, for the J 9, J 12 and J 24 treatments $0 \mathrm{~h}, 1 \mathrm{~h}$ and $2 \mathrm{~h}$ post-transport compared with baseline, whereas the J 18 treatment had greater glucose concentrations $(\mathrm{P}<0.05)$ at $0 \mathrm{~h}$ and $1 \mathrm{~h}$ post-transport.

NEFA concentrations were greater in non-transported control animals (J 0$)$ at $0 \mathrm{~h}, 1 \mathrm{~h}, 2$, $\mathrm{h}$ and $24 \mathrm{~h}$ compared with baseline (Table 6). There were transient increases $(\mathrm{P}<0.05)$ in NEFA concentrations for the $\mathrm{J} 6$ treatment at $12 \mathrm{~h}$ post-transport whereas the J 9 and $\mathrm{J} 12$ treatments had lower $(\mathrm{P}<0.05)$ NEFA concentrations at $1 \mathrm{~h}$ to $6 \mathrm{~h}$ post-transport. Following transportation of animals for $18 \mathrm{~h}$ (J 18), NEFA concentrations were greater $(\mathrm{P}<0.05)$ at $0 \mathrm{~h}$ and $24 \mathrm{~h}$ whereas animals transported for $24 \mathrm{~h}$ had greater concentrations than baseline at $0 \mathrm{~h}$, $1 \mathrm{~h}$ and $24 \mathrm{~h}$ post-transport.

WBC numbers were greater $(\mathrm{P}<0.05)$ than baseline at $4 \mathrm{~h}$ to $24 \mathrm{~h}$ (Table 7 ) in non-transported control animals (J 0). There were transient increases in WBC number for the $\mathrm{J} 6$ treatment at $0 \mathrm{~h}$ to $24 \mathrm{~h}$ post-transport and for the J 9 and J 18 treatments at $0 \mathrm{~h}$ to $12 \mathrm{~h}$ post-transport, while the J 12 and J 24 treatments had greater $(\mathrm{P}<$ $0.05) \mathrm{WBC}$ number at $0 \mathrm{~h}$ to $24 \mathrm{~h}$ post-transport.

The $\mathrm{N}: \mathrm{L}$ ratio was greater $(\mathrm{P}<0.05)$ than baseline at $12 \mathrm{~h}$ (Table 7) in non-transported control animals (J 0). There were transient increases $(\mathrm{P}<0.05)$ in $\mathrm{N}$ :L ratios for the $\mathrm{J} 6$ treatment at $0 \mathrm{~h}$ to $4 \mathrm{~h}$ post-transport; the $\mathrm{J} 9$ treatment at $0 \mathrm{~h}$ to $8 \mathrm{~h}$ post-transport; the J 12 treatment at $0 \mathrm{~h}$ to $2 \mathrm{~h}$ and 8 and $12 \mathrm{~h}$ post-transport; the J 18 treatment at $1 \mathrm{~h}$ to $4 \mathrm{~h}$, and $8 \mathrm{~h}$ and $12 \mathrm{~h}$ posttransport and the J 24 treatment at $0 \mathrm{~h}, 2 \mathrm{~h}, 4 \mathrm{~h}$ and $12 \mathrm{~h}$ post-transport.

In non-transported control animals, lymphocyte percentage was lower $(\mathrm{P}<0.05)$ and neutrophil percentage was greater $(\mathrm{P}<0.05)$ than baseline at $6 \mathrm{~h}$ to $24 \mathrm{~h}$ following the end of the $24 \mathrm{~h}$ experimental period (Table 8). Animals transported for 6 (J 6), 9 (J 9), 12 (J 12), 18 (J 18) and $24 \mathrm{~h}(\mathrm{~J} 24)$ had lower $(\mathrm{P}<0.05)$ lymphocyte percentage and greater $(\mathrm{P}<0.05)$ neutrophil percentage than baseline at $0 \mathrm{~h}$ to $24 \mathrm{~h}$ post-transport. 
Table 7 Treatment means ( $n=12$ animals per treatment) with $\mathrm{SE}^{1}$ for white blood cell (WBC) number and the neutrophil:lymphocyte ( $\mathrm{N}: \mathrm{L})$ ratio pre $(-0.25 \mathrm{~h})$ and post-transport $(0,1,2,4,6,8,12$ and $24 \mathrm{~h})$

\begin{tabular}{|c|c|c|c|c|c|c|c|c|c|c|c|}
\hline Transport & $-0.25 \mathrm{~h}$ pre & $0 \mathrm{~h}$ post & $1 \mathrm{~h}$ post & $2 \mathrm{~h}$ post & $4 \mathrm{~h}$ post & $6 \mathrm{~h}$ post & $8 \mathrm{~h}$ post & $12 \mathrm{~h}$ post & $24 \mathrm{~h}$ post & $\mathrm{SE}^{1}$ & Effect of time ${ }^{2}$ \\
\hline \multicolumn{12}{|c|}{$\overline{W B C}\left(\times 10^{3} / \mu \mathrm{L}\right)$} \\
\hline J 0 & $9.2^{\mathrm{a}}$ & $9.1^{\mathrm{ab}}$ & $8.9^{\mathrm{ab}}$ & $9.8^{\mathrm{ab}}$ & $11.5^{\mathrm{b}}$ & $11.3^{\mathrm{b}}$ & $11.9^{b}$ & $12.6^{\mathrm{b}}$ & $12.2^{b}$ & 0.72 & $P=0.0003$ \\
\hline 16 & $8.9^{\mathrm{a}}$ & $14.7^{\mathrm{b}}$ & $15.2^{\mathrm{b}}$ & $15.1^{\mathrm{b}}$ & $14.4^{\mathrm{b}}$ & $14.5^{\mathrm{b}}$ & $14.2^{\mathrm{b}}$ & $13.5^{\mathrm{b}}$ & $11.8^{\mathrm{b}}$ & 0.07 & $P=0.001$ \\
\hline J 9 & $9.7^{\mathrm{a}}$ & $15.3^{\mathrm{b}}$ & $15.7^{\mathrm{b}}$ & $15.5^{\mathrm{b}}$ & $15.2^{\mathrm{b}}$ & $15.1^{b}$ & $15.1^{\mathrm{b}}$ & $13.8^{\mathrm{b}}$ & $11.7^{\mathrm{ab}}$ & 0.79 & $P=0.0001$ \\
\hline $\mathrm{J} 12$ & $9.3^{\mathrm{a}}$ & $12.7^{\mathrm{ab}}$ & $13.2^{\mathrm{b}}$ & $13.3^{b}$ & $13.2^{\mathrm{b}}$ & $13.3^{b}$ & $13.7^{\mathrm{b}}$ & $12.5^{\mathrm{b}}$ & $11.6^{\mathrm{b}}$ & 0.76 & $P=0.003$ \\
\hline J 18 & $10.4^{\mathrm{a}}$ & $13.1^{\mathrm{b}}$ & $13.1^{\mathrm{b}}$ & $13.4^{\mathrm{b}}$ & $13.1^{\mathrm{b}}$ & $14.0^{b}$ & $14.1^{b}$ & $13.9^{b}$ & $12.2^{\mathrm{ab}}$ & 0.65 & $P=0.004$ \\
\hline $\mathrm{J} 24$ & $10.4^{\mathrm{a}}$ & $11.9^{\mathrm{b}}$ & $12.7^{b}$ & $12.2^{\mathrm{b}}$ & $12.6^{\mathrm{b}}$ & $13.3^{b}$ & $14.1^{\mathrm{b}}$ & $13.4^{b}$ & $14.0^{\mathrm{b}}$ & 0.99 & $P=0.20$ \\
\hline \multicolumn{12}{|l|}{ N:L ratio } \\
\hline J 0 & $0.35^{\mathrm{a}}$ & $0.28^{\mathrm{ab}}$ & $0.32^{\mathrm{ab}}$ & $0.40^{\mathrm{ab}}$ & $0.51^{\mathrm{ab}}$ & $0.68^{\mathrm{ab}}$ & $0.77^{\mathrm{ab}}$ & $1.35^{\mathrm{b}}$ & $0.57^{\mathrm{ab}}$ & 0.09 & $P=0.0001$ \\
\hline 16 & $0.62^{\mathrm{a}}$ & $1.30^{\mathrm{b}}$ & $1.27^{\mathrm{b}}$ & $1.33^{\mathrm{b}}$ & $1.37^{\mathrm{b}}$ & $1.20^{\mathrm{ab}}$ & $1.15^{\mathrm{ab}}$ & $0.78^{\mathrm{ab}}$ & $0.76^{\mathrm{ab}}$ & 0.05 & $P=0.0001$ \\
\hline J 9 & $0.44^{\mathrm{a}}$ & $1.65^{b}$ & $1.63^{\mathrm{b}}$ & $1.53^{\mathrm{b}}$ & $1.13^{\mathrm{b}}$ & $1.09^{b}$ & $1.12^{b}$ & $0.97^{\mathrm{ab}}$ & $0.85^{\mathrm{ab}}$ & 0.10 & $P=0.0001$ \\
\hline J 12 & $0.31^{\mathrm{a}}$ & $1.15^{b}$ & $1.17^{\mathrm{b}}$ & $1.12^{\mathrm{b}}$ & $0.72^{\mathrm{ab}}$ & $0.73^{\mathrm{ab}}$ & $0.99^{b}$ & $0.93^{b}$ & $0.58^{\mathrm{ab}}$ & 0.08 & $P=0.0001$ \\
\hline J 18 & $0.44^{\mathrm{a}}$ & $0.95^{\mathrm{ab}}$ & $1.07^{\mathrm{b}}$ & $1.06^{\mathrm{b}}$ & $1.01^{\mathrm{b}}$ & $0.92^{\mathrm{ab}}$ & $1.36^{\mathrm{b}}$ & $1.05^{\mathrm{b}}$ & $0.68^{\mathrm{ab}}$ & 0.11 & $P=0.0001$ \\
\hline $\mathrm{J} 24$ & $0.43^{\mathrm{a}}$ & $0.99^{b}$ & $0.81^{\mathrm{ab}}$ & $1.08^{\mathrm{b}}$ & $1.10^{\mathrm{b}}$ & $0.77^{\mathrm{ab}}$ & $0.83^{\mathrm{ab}}$ & $0.92^{\mathrm{b}}$ & $0.84^{\mathrm{ab}}$ & 0.14 & $P=0.0001$ \\
\hline
\end{tabular}

Control = J 0 not transported; animals transported for journey (J) durations of 6, 9, 12, 18 and 24 hours (h) by road at a stocking density of 1.02 m ${ }^{2}$ per animal. ${ }^{\mathrm{a}, \mathrm{b}}$, within a row, means differ from pre-transport baseline $\left(-0.25 \mathrm{~h}\right.$ pre-transport) at $\mathrm{P}<0.05$. $^{1}$ Standard error (SE). ${ }^{2}$ Effect of time (exact $\mathrm{P}$ value).

RBC numbers were lower $(\mathrm{P}<0.05)$ than baseline in non-transported control animals at $0 \mathrm{~h}$ to $24 \mathrm{~h}$ relative to the end of the first $24 \mathrm{~h}$ experimental period (Table 9). There were no differences $(\mathrm{P}>0.05)$ in $\mathrm{RBC}$ numbers in the J 6, J 9, J 12, J 18 and J 24 treatments following transport compared with baseline values.

Haemoglobin $(\mathrm{Hgb})$ concentrations were lower $(\mathrm{P}<$ $0.05)$ at $0 \mathrm{~h}$ to $24 \mathrm{~h}$ in non-transported control animals compared with baseline (Table 9). There were no differences $(\mathrm{P}>0.05)$ in $\mathrm{Hgb}$ concentrations for J 6, J 9, J 18 and J 24 treatment groups following transport while animals transported for $12 \mathrm{~h}$ had lower Hgb concentrations $(\mathrm{P}<0.05)$ than baseline at $24 \mathrm{~h}$ post-transport.
Haematocrit \% was lower $(\mathrm{P}<0.05)$ at $0 \mathrm{~h}$ to $24 \mathrm{~h}$ post-transport (Table 9) compared with baseline in nontransported control animals. Animals transported for 6 $\mathrm{h}(\mathrm{J} 6)$ and $12 \mathrm{~h}(\mathrm{~J} 12)$ had lower $(\mathrm{P}<0.05)$ haematocrit $\%$ than baseline at $24 \mathrm{~h}$ post-transport. Post-transport there were no differences $(\mathrm{P}>0.05)$ in haematocrit \% in J 9, J 18 and J 24 treatments compared with baseline values.

There was no difference $(\mathrm{P}>0.05)$ in mean corpuscular volume (MCV) and $\mathrm{MCHC}$ following transport (data not shown). Platelet numbers were lower $(\mathrm{P}<0.05)$ at $0 \mathrm{~h}$ (mean (s.d.) $424.3(140.4) \times 10^{3}$ cells $\left./ \mu \mathrm{L}\right)$ in nontransported control animals compared with $4 \mathrm{~h}$ (mean

Table 8 Treatment means ( $\mathrm{n}=12$ animals per treatment) with $\mathrm{SE}^{1}$ for lymphocyte and neutrophil percentage (\%) pre $(-0.25 \mathrm{~h})$ and post-transport $(0,1,2,4,6,8,12$ and $24 \mathrm{~h})$

\begin{tabular}{|c|c|c|c|c|c|c|c|c|c|c|c|}
\hline Transport & $-0.25 \mathrm{~h}$ pre & $0 \mathrm{~h}$ post & $1 \mathrm{~h}$ post & $2 \mathrm{~h}$ post & $4 \mathrm{~h}$ post & $6 \mathrm{~h}$ post & $8 \mathrm{~h}$ post & $12 \mathrm{~h}$ post & $24 \mathrm{~h}$ post & $\mathrm{SE}^{1}$ & Effect of time ${ }^{2}$ \\
\hline \multicolumn{12}{|c|}{ Lymphocyte (\%) } \\
\hline $\mathrm{J} O$ & $73.2^{\mathrm{a}}$ & $77.8^{\mathrm{ab}}$ & $74.5^{\mathrm{ab}}$ & $71.9^{\mathrm{ab}}$ & $68.3^{a b}$ & $62.0^{b}$ & $58.3^{b}$ & $44.8^{b}$ & $63.8^{b}$ & 3.35 & $P=0.0001$ \\
\hline 16 & $65.2^{a}$ & $43.6^{b}$ & $46.7^{b}$ & $43.1^{b}$ & $42.9^{b}$ & $46.3^{b}$ & $47.2^{b}$ & $56.0^{b}$ & $57.3^{b}$ & 2.49 & $P=0.0001$ \\
\hline J 9 & $65.9^{a}$ & $39.8^{b}$ & $41.4^{\mathrm{b}}$ & $41.8^{b}$ & $47.7^{b}$ & $48.4^{b}$ & $47.8^{b}$ & $52.0^{b}$ & $53.7^{b}$ & 3.02 & $P=0.0001$ \\
\hline 12 & $74.6^{\mathrm{a}}$ & $49.5^{b}$ & $46.5^{b}$ & $49.0^{b}$ & $59.5^{b}$ & $60.0^{b}$ & $51.0^{b}$ & $51.6^{b}$ & $63.6^{b}$ & 2.99 & $P=0.0001$ \\
\hline J 18 & $68.4^{a}$ & $52.8^{b}$ & $48.9^{b}$ & $50.3^{b}$ & $50.8^{b}$ & $51.8^{b}$ & $43.5^{b}$ & $49.6^{b}$ & $59.8^{b}$ & 2.94 & $P=0.0001$ \\
\hline $\mathrm{J} 24$ & $69.4^{\mathrm{a}}$ & $50.8^{b}$ & $56.6^{\mathrm{b}}$ & $49.2^{b}$ & $47.2^{b}$ & $56.1^{b}$ & $53.8^{b}$ & $52.2^{b}$ & $56.3^{b}$ & 2.79 & $P=0.0001$ \\
\hline \multicolumn{12}{|c|}{ Neutrophil (\%) } \\
\hline $\mathrm{J} 0$ & $24.1^{a}$ & $19.8^{b}$ & $23.1^{b}$ & $26.6^{b}$ & $29.9^{b}$ & $36.1^{b}$ & $40.3^{b}$ & $54.1^{b}$ & $34.8^{b}$ & 3.31 & $P=0.0001$ \\
\hline J 6 & $30.8^{a}$ & $55.2^{b}$ & $55.5^{\mathrm{ab}}$ & $55.6^{\mathrm{ab}}$ & $56.1^{\mathrm{ab}}$ & $52.8^{b}$ & $51.3^{b}$ & $42.3^{b}$ & $42.8^{b}$ & 2.56 & $P=0.0001$ \\
\hline J 9 & $25.6^{a}$ & $60.1^{b}$ & $59.1^{b}$ & $57.9^{b}$ & $51.8^{b}$ & $51.0^{\mathrm{b}}$ & $50.5^{b}$ & $45.8^{b}$ & $42.8^{b}$ & 2.74 & $P=0.0001$ \\
\hline J 12 & $22.9^{a}$ & $48.6^{b}$ & $52.8^{b}$ & $50.8^{b}$ & $39.9^{b}$ & $38.7^{b}$ & $47.5^{b}$ & $46.4^{b}$ & $34.7^{b}$ & 3.04 & $P=0.0001$ \\
\hline J 18 & $28.2^{a}$ & $46.1^{b}$ & $50.4^{b}$ & $48.7^{b}$ & $48.7^{b}$ & $44.8^{b}$ & $54.5^{b}$ & $46.7^{b}$ & $36.8^{b}$ & 2.96 & $P=0.0001$ \\
\hline J 24 & $28.9^{a}$ & $48.7^{b}$ & $42.7^{b}$ & $49.0^{b}$ & $50.2^{b}$ & $41.3^{b}$ & $42.3^{b}$ & $43.6^{b}$ & $41.8^{b}$ & 2.90 & $P=0.0001$ \\
\hline
\end{tabular}

Control = J 0 not transported; animals transported for journey (J) durations of 6, 9, 12, 18 and 24 hours (h) by road at a stocking density of 1.02 mer animal. ${ }^{a}, b_{\text {, }}$ within a row, means differ from pre-transport baseline $\left(-0.25 \mathrm{~h}\right.$ pre-transport) at $\mathrm{P}<0.05$. ${ }^{1}$ Standard error (SE). ${ }^{2}$ Effect of time (exact $\mathrm{P}$ value). 
Table 9 Treatment means with $\mathrm{SE}^{1}$ for red blood cell (RBC) number, whole blood haemoglobin (Hgb) concentrations, and haematocrit (\%) pre (-0.25 h) and post-transport (0, 1, 2, 4, 6, 8, 12 and $24 \mathrm{~h})$

\begin{tabular}{|c|c|c|c|c|c|c|c|c|c|c|c|}
\hline Transport & $-0.25 \mathrm{~h}$ pre & $0 \mathrm{~h}$ post & $1 \mathrm{~h}$ post & $2 \mathrm{~h}$ post & $4 \mathrm{~h}$ post & $6 \mathrm{~h}$ post & $8 \mathrm{~h}$ post & $12 \mathrm{~h}$ post & $24 \mathrm{~h}$ post & $\mathrm{SE}^{1}$ & Effect of time ${ }^{2}$ \\
\hline \multicolumn{12}{|c|}{$\overline{\mathrm{RBC}\left(\times 10^{6} / \mu \mathrm{L}\right)}$} \\
\hline J 0 & $10.0^{a}$ & $9.3^{b}$ & $8.8^{\mathrm{b}}$ & $8.8^{\mathrm{b}}$ & $9.1^{b}$ & $9.3^{b}$ & $8.8^{b}$ & $9.1^{b}$ & $9.0^{\mathrm{b}}$ & 0.25 & $P=0.02$ \\
\hline 16 & 9.4 & 9.5 & 9.5 & 9.4 & 9.2 & 9.2 & 9.0 & 9.1 & 8.7 & 0.30 & $P=0.58$ \\
\hline J 9 & 9.3 & 9.6 & 9.6 & 9.5 & 9.3 & 9.2 & 9.3 & 9.4 & 9.1 & 0.40 & $P=0.993$ \\
\hline J 12 & 9.6 & 9.5 & 9.7 & 9.8 & 9.6 & 9.4 & 9.4 & 9.4 & 9.0 & 0.30 & $P=0.823$ \\
\hline J 18 & 9.9 & 9.8 & 9.8 & 9.8 & 10.1 & 9.8 & 9.9 & 10.0 & 9.2 & 0.29 & $P=0.75$ \\
\hline J 24 & 8.8 & 8.9 & 9.1 & 9.2 & 9.0 & 8.7 & 8.8 & 8.9 & 8.7 & 0.25 & $P=0.81$ \\
\hline \multicolumn{12}{|l|}{$\mathrm{Hgb}(\mathrm{g} / \mathrm{dL})$} \\
\hline J 0 & $11.2^{\mathrm{a}}$ & $10.4^{b}$ & $10.0^{\mathrm{b}}$ & $9.8^{\mathrm{b}}$ & $10.1^{\mathrm{b}}$ & $10.2^{b}$ & $9.7^{b}$ & $9.9^{b}$ & $9.9^{\mathrm{b}}$ & 0.28 & $P=0.01$ \\
\hline 16 & 10.0 & 10.0 & 10.0 & 10.0 & 9.7 & 9.6 & 9.5 & 9.6 & 9.3 & 0.24 & $P=0.35$ \\
\hline J 9 & 9.5 & 9.5 & 9.7 & 9.5 & 9.3 & 9.3 & 9.2 & 9.6 & 9.2 & 0.26 & $P=0.887$ \\
\hline J 12 & $9.9^{\mathrm{a}}$ & $9.8^{\mathrm{ab}}$ & $10.1^{\mathrm{ab}}$ & $10.1^{\mathrm{ab}}$ & $9.9^{\mathrm{ab}}$ & $9.7^{\mathrm{ab}}$ & $9.9^{\mathrm{ab}}$ & $9.8^{\mathrm{ab}}$ & $9.3^{b}$ & 0.18 & $P=0.120$ \\
\hline J 18 & 10.1 & 10.0 & 10.1 & 10.0 & 10.1 & 9.9 & 9.8 & 10.1 & 9.1 & 0.26 & $P=0.15$ \\
\hline J 24 & 9.0 & 9.1 & 9.4 & 9.5 & 9.2 & 9.2 & 9.3 & 9.3 & 9.3 & 0.22 & $P=0.86$ \\
\hline \multicolumn{12}{|c|}{ Haematocrit (\%) } \\
\hline J 0 & $33.9^{\mathrm{a}}$ & $31.7^{\mathrm{b}}$ & $29.8^{\mathrm{b}}$ & $29.5^{\mathrm{b}}$ & $30.3^{b}$ & $30.3^{b}$ & $29.1^{b}$ & $30.2^{\mathrm{b}}$ & $29.7^{b}$ & 0.94 & $P=0.02$ \\
\hline 16 & $30.4^{\mathrm{a}}$ & $31.2^{\mathrm{ab}}$ & $31.1^{\mathrm{ab}}$ & $30.8^{\mathrm{ab}}$ & $29.9^{\mathrm{ab}}$ & $29.7^{\mathrm{ab}}$ & $29.2^{\mathrm{ab}}$ & $29.5^{\mathrm{ab}}$ & $27.6^{\mathrm{b}}$ & 0.78 & $P=0.05$ \\
\hline J 9 & 28.5 & 29.7 & 29.8 & 29.2 & 28.7 & 28.3 & 28.5 & 28.9 & 27.7 & 0.94 & $P=0.87$ \\
\hline J 12 & $29.9^{\mathrm{a}}$ & $30.0^{\mathrm{ab}}$ & $30.4^{\mathrm{ab}}$ & $30.4^{\mathrm{ab}}$ & $29.7^{\mathrm{ab}}$ & $29.1^{\mathrm{ab}}$ & $29.1^{\mathrm{ab}}$ & $29.0^{\mathrm{ab}}$ & $27.9^{b}$ & 0.66 & $P=0.20$ \\
\hline J 18 & 30.1 & 29.9 & 30.1 & 29.5 & 30.6 & 29.8 & 29.8 & 30.5 & 28.0 & 0.88 & $P=0.73$ \\
\hline J 24 & 27.2 & 27.4 & 27.8 & 28.3 & 27.3 & 27.1 & 27.1 & 27.6 & 27.0 & 0.65 & $P=0.93$ \\
\hline
\end{tabular}

Control = J 0 not transported; animals transported for journey $(\mathrm{J})$ durations of $6,9,12,18$ and 24 hours (h) by road at a stocking density of 1.02 m ${ }^{2}$ per animal. ${ }^{a}, b_{\text {, }}$ within a row, means differ from pre-transport baseline $\left(-0.25 \mathrm{~h}\right.$ pre-transport) at $\mathrm{P}<0.05$. ${ }^{1}$ Standard error (SE). ${ }^{2}$ Effect of time (exact $\mathrm{P}$ value).

(s.d.) $536.7(135.4) \times 10^{3}$ cells $\left./ \mu \mathrm{L}\right), 6 \mathrm{~h}$ (mean (s.d.) 561.3 $(155.8) \times 10^{3}$ cells $\left./ \mu \mathrm{L}\right)$ and $12 \mathrm{~h}$ (mean 539.9 (s.d.) $\left.(124.0) \times 10^{3} \mathrm{cells} / \mu \mathrm{L}\right)$ and values returned to baseline at $24 \mathrm{~h}$. Platelet numbers were not different $(\mathrm{P}>0.05)$ post-transport for the J 6 to J 24 treatments (data not shown).

\section{Discussion}

In the present study, the series of transport journeys (6 $\mathrm{h}$ to $24 \mathrm{~h}$ ) by roads showed that transportation of Charolais beef bulls affected live weight, haematological and physiological measurements of metabolism and inflammation. The biological measures which were most sensitive to the stress of transport, on journeys of $6 \mathrm{~h}$ to $24 \mathrm{~h}$ duration, were total protein, urea, $\mathrm{BHB}$, glucose, NEFA, the acute phase protein (haptoglobin) and haematological variables (including WBC number, neutrophil and lymphocyte \%). This was not unexpected as we reported similar responses in young bulls that were subjected to $8 \mathrm{~h}$ transport [4]. However, it also flags a cautionary note when drawing conclusions based on a single cohort of animals that to increase the probability of statistically significant results when measuring other variables (for example RBC number, albumin, globulin, and haemoglobin and creatine kinase activity) it may be necessary to increase the group size per treatment.
The present experiment was designed to minimize any possible bias that would affect the study, however, we accept that it is not possible to exclude all bias since all studies are affected by some degree of bias. In the present study, the bulls were habituated to housing for 100 days pre-transport and were fed the same diet. All of the bulls were naïve to transport and each journey was carried out singly over a 6 -week period. For each journey, 12 bulls were assigned to two pens on the transporter with 6 animals per pen and each journey was made by the same driver using similar road conditions. The bulls were blood sampled by the same person and the same chute was used at each experimental blood collection time point. In the statistical analysis of the data, animal was the experimental or measurement unit.

The changes in live weight post-transport were transitory and may be attributed to a loss of gut-fill over the journeys and possibly due to mild dehydration, urination and fasting during the longer journeys [15-17,20,21]. The loss in live weight in control animals in the present study may be attributed to the change in diet, as silage and concentrates were withdrawn and animals had access to hay and water only for $24 \mathrm{~h}$ corresponding to the longest duration of transport. Rectal temperature was not changed during each of the respective transport journeys indicating that there was no clinical infection induced by transport and no evidence of clinical disease. 
Although rectal temperature was not changed, it is a well known indicator of an inflammatory response to infection in newly arrived feedlot calves [20]. The lack of an effect of transport on rectal body temperature may be related to the ambient temperature since animals would not have been exposed to extreme range of temperatures during the present series of journeys.

The development of electrolyte and acid-base imbalances has been reported in extended transport journeys where fasting has exceeded 2 days or more [22]. In the present study, transportation had transient effects on metabolism as demonstrated by significant changes in the plasma concentrations of total protein, urea, $\beta \mathrm{HB}$, NEFA and glucose. Total protein concentrations increased with journey duration in transported animals, however, by $12 \mathrm{~h}$ post-transport concentrations had returned to pre-transport baseline levels. Transport stress has been reported to cause dehydration and may manifest itself as a hyperproteinemia [23]. The changes in protein concentrations reported in the present study post-transport are more likely the result of metabolic compensation for a mild metabolic acidosis secondary to water loss and feed deprivation during transport. These findings suggest that pre-transport mixing and transportation alters protein metabolism. Metabolic variables of protein, energy, and mineral metabolism in cattle as well as rumen function have been examined following transportation. Changes in circulating total protein, albumin, and urea, have been reported to increase following transportation $[3,24]$. Changes in energy metabolism as evidenced by an increases in blood glucose $[1,16,17]$, lactate dehydrogenase, glutamic pyruvic transaminase, and glutamic oxalacetic transaminase [24], decreases in $\beta \mathrm{HB}$ [3], increased haematocrit \% and plasma corticosteroid concentration [25] have been reported. When the body prepares to react to a potentially stressful situation an increase in energy metabolism may be precipitated [26].

Increases in plasma glucose concentrations are mainly due to glycogenolysis associated with the increase in circulating catecholamines and glucocorticoids which are released during the stress of transport [27]. Glucose levels returned to baseline in all treatments compared with baseline within $4 \mathrm{~h}$ of transport. Urea, NEFA and $\beta \mathrm{HB}$ concentrations were elevated in control and all transported animals and concentration remained greater than baseline for animals transported on journey durations ranging from $0 \mathrm{~h}$ to $24 \mathrm{~h}$. Urea concentrations had returned to pre-transport baseline values by $24 \mathrm{~h}$ posttransport in all animals.

Physiological processes, such as the sleep-wake cycles, locomotor activity, body temperature, hormone secretion, and metabolism, are under the control of circadian clocks and are influenced by stress. Circadian control of glucose metabolism was recognized from early studies demonstrating variation in glucose tolerance and insulin action across the day $[28,29]$. Increased energy metabolism is a hallmark of the stress response as the body prepares to react to a potentially stressful situation. We have previously reported increases in several of these protein metabolites in response to transportation $[3,4,17]$. These differences may be due to a number of factors including the duration of the journey and that animals did not have access to feed during transportation. Additionally, increased circulating $\mathrm{CK}$ is an indication of muscular activity and or/bruising and is often measured in transported cattle as a measure of bruising [5]. Creatine and phosphocreatine are major intracellular solutes in muscle cells. Increases in plasma CK activity after different transport journeys have been described by different authors [16,19,30]. A direct relationship between the duration of transport and the rise in the activity of the enzyme has been reported [19]. Fasting has also been reported to increase the activity of the enzyme, and the rise could be masked by the high values obtained after transport [31]. In the present study, CK activities returned to pre-treatment baseline values within $12 \mathrm{~h}$ for all transported animals. Interestingly, control animals in the present study had elevated CK activity while the magnitude of the changes were small the return to baseline was rapid. Circulating creatine kinase activity is often measured in transported catthe as a measure of bruising [32], indicating that the bulls in the current study may have experienced some physical stress.

Changes in acute phase protein concentrations during transportation have been reported but the results are variable. Haptoglobin, an acute phase protein, is released by hepatocytes and mediate the inflammatory response to injury, trauma, or infection [33]. The presence of acute phase proteins in the circulation may be an excellent biomarker of inflammation as they are readily measurable in serum or plasma and may even discriminate between acute and chronic inflammation in cattle [34]. Acute phase proteins are present in very low concentrations in plasma and increase in concentration following tissue injury and inflammation $[35,36]$. In the present study haptoglobin concentrations were increased relative to the baseline in control and transported animals up to $24 \mathrm{~h}$ post-transport, with the exception of the animals transported for $6 \mathrm{~h}$. Serum haptoglobin was reported to be elevated in calves transported for 2 days and levels were negatively correlated with lymphocyte function [37]. In a separate study transporting bulls at different stocking densities, plasma haptoglobin concentrations were unchanged, while plasma fibrinogen levels were reduced $[3,4]$. Fibrinogen, ceruloplasmin, serum amyloid-A, and $\alpha$-acid glycoprotein were assayed in the 
plasma of transported and commingled calves and found to be increased post-transportation; however, haptoglobin concentrations were greater in non-transported versus transported calves [20].

Alterations in immunity are of great importance following transportation stress as these alterations are thought to be associated with increased incidence and severity of respiratory diseases. Many measures of immunological changes relate to immune cell numbers in the blood. Similar to the findings of the present study, most studies observe a leukocytosis that is marked by neutrophilia, which may occur in conjunction with a decrease in the number of other cells (lymphopaenia, eosinopaenia) $[9,10,38]$. Changes in the haematological responses of cattle to transport have been reported with increases in RBC number, haematocrit percentage and haemoglobin concentration following transportation of steers $[17,39]$. In the present study, all transported animals had greater neutrophil percentage and lower lymphocyte percentage post-transport. Haemoglobin concentrations and RBC numbers were within normal blood referenced ranges [40-43]. The neutrophilia observed in control animals is most likely due to the effect of stress related to the mixing and the handling procedures. Blood lymphocytes contain concentrations of glucocorticoid and adrenergic receptors [44], which are down-regulated in response to stress [45] and suggests that alterations in the blood cell composition of leukocytes may have an important role in the responsiveness of the immune system when stress challenged. There was no major change in haematocrit $\%$ compared with baseline in animals transported for 6 $\mathrm{h}$ to $24 \mathrm{~h}$. Animals in the present study had ad libitum access to water on the transporter and they received the last feeding immediately before loading and these factors may have prevented the animals from showing signs of dehydration. Elevated haematocrit \% has been reported following transport in association with greater erythrocyte counts in the circulation $[17,25,46,47]$ and a significant increase in haematocrit values indicates mainly dehydration.

Measures of immunological changes relate to immune cell numbers in the blood and immune cell function. A number of studies have reported leukocytosis that is marked by neutrophilia, and which may be present with a decrease in the number of other cells (lymphopaenia, eosinopaenia) $[8,10,17]$. Bovine alveolar macrophages, isolated from bronchoalveolar lavage (BAL) fluid, have a reduced respiratory burst function after $4 \mathrm{~h}$ of transportation [48]. The respiratory burst function is necessary to produce reactive oxygen species that are toxic to phagocytosed pathogens, and these results may represent impaired lung defence. In contrast, enhanced respiratory burst activity has been found in neutrophils of transported calves [10]. Apoptosis of neutrophils in combination with increased migratory capacity in dairy cows have been reported after $4 \mathrm{~h}$ of transportation [49]. The normal referenced ranges for differential counts, neutrophils are in the range 15-45 [40,50]. Within the range of transport times analysed, there were no significant changes in $\mathrm{MCV}$ and $\mathrm{MCHC}$ values. In the present study, the changes in the composition of the blood cell variables reflect the physiological response of the bulls to the stress of mixing, fasting and/or transportation.

In the current study, several measures of physiological, metabolic and haematological variables were investigated in the plasma of bulls subjected to transport journeys ranging from 6 to $24 \mathrm{~h}$ durations. It is evident that transportation of bulls has effects on biomarkers of metabolism as demonstrated by significant changes in the plasma concentrations of protein, glucose and NEFA. Additionally, circulating creatine kinase activity is a useful measure which is often monitored in transported cattle as a measure of bruising. Changes in CK activity indicate that the bulls in the current study may have experienced some muscle damage and physical stress, particularly after the longer duration journeys. The acute phase protein, haptoglobin, is a useful biomarker of inflammation and together with changes in haematological cellular variables would suggest a proinflammatory state during transportation stress. The pronounced neutrophilia and lymphopenia following transportation observed in this study are in agreement with previously reported findings following a variety of stressors, including transport stress $[8,10,17]$. Taken together, these results indicate that transportation stress alters physiological measures of metabolism and haematology. Thus, a profile or pattern of multiple physiological, metabolic and haematological variables may provide the most effective marker of altered homeostasis to allow an assessment of an animal's response to transport.

\section{Conclusions}

Conditional on the statistical power of the present study and assumptions about meaningful indicators and effect of size, there were both similarities and differences between control and transported animals, however the differences did not appear sufficiently large or prolonged over the duration of the study. This is a single transport study for each journey duration and most of the physiological, metabolic and haematological variables which changed as a consequence of transport had recovered to baseline values within $24 \mathrm{~h}$ of journey completion. Nontransported control animals showed similar biological effects on the physiological and haematological variables to transported animals. 
An increased understanding of the mechanism of stress and physiological adaptation induced in animals by mixing, handling and transport will lead to a greater understanding of transportation stress. Additionally, the effective recovery of the animals after 6-24 hours transport can lead to an important implication to recommend a proper lairage time when animals are transported by road. The results of this study indicate that the provision of a rest period of up to 24 hours post-transport with animals having access to feed and water should be optimal for animals to recover to their physiological pre-transport baseline.

\section{Methods}

\section{Care of animals}

This study was conducted at Teagasc, Grange Beef Research Centre, Dunsany, County Meath, Ireland. All animal procedures performed in this study were conducted under experimental licence from the Irish Department of Health and Children in accordance with the Cruelty to Animals Act 1876 and the European Communities (Amendment of Cruelty to Animals Act 1876) Regulation 2002 and 2005.

\section{Animal diets and composition}

Seventy two Charolais bulls (mean weight (s.d.) 367 (35) $\mathrm{kg}$ ) had ad libitum access to grass silage (in vitro DM digestibility $=762 \mathrm{~g} / \mathrm{kg}$ ), supplemented with $2.0 \mathrm{~kg}$ (as fed) barley/soybean concentrate (crude protein $=114.5$ kg DM) per animal per day at Teagasc, Grange, Beef Research Centre, Dunsany, Co. Meath. The animals were managed and housed 6/pen in a slatted floor shed at a space allowance of $2.5 \mathrm{~m}^{2} / \mathrm{head} /$ animal for a 100 day winter period prior to transport and are representative of the type of animals that are commonly transported under commercial conditions. Animals had free access to water which was available through nipple drinkers in their home pens.

\section{Transport vehicle and environmental conditions}

The study was conducted in Spring over a 6 week period from the $18^{\text {th }}$ of February to the $29^{\text {th }}$ of March. All journeys were made at the same starting time $(8: 00 \mathrm{~h})$ and with the same transporter, transport conditions and driver. A total of 5 separate transport journeys were made of duration 6, 9, 12, 18 and $24 \mathrm{~h}$. All animals were naïve to transport. On the morning of each journey, the animals were loaded at 8:00 $\mathrm{h}$ into two fan-ventilated pens ( $n=6$ animals/pen) on the lower deck of an air suspension articulated transporter at a stocking density of $1.02 \mathrm{~m}^{2}$ per animal, and transported by road. The floor of the pens on the transporter was deep bedded with cereal straw for each journey. The animals had access to hay and water was available through nipple drinkers on the transporter, similar to the type that the bulls were accustomed to in their home pens. Bulls travelled for $6 \mathrm{~h}(280 \mathrm{~km}), 9 \mathrm{~h}(435 \mathrm{~km}), 12 \mathrm{~h}$ $(582 \mathrm{~km}), 18 \mathrm{~h}(902 \mathrm{~km})$ and $24 \mathrm{~h}(1192 \mathrm{~km})$ and during each journey, transport was by primary (30\% of the journey) and secondary (70\% of the journey) roads and different road types and surfaces were encountered, respectively. The transporter was fitted with sensors, located $1.2 \mathrm{~m}$ above the floor of each pen on the transporter, for measuring ambient temperature $\left({ }^{\circ} \mathrm{C}\right)$, relative humidity ( $\mathrm{RH} ; \%)$, carbon dioxide $\left(\mathrm{CO}_{2}\right.$; $\left.\mathrm{ppm}\right)$, hydrogen sulphide $\left(\mathrm{H}_{2} \mathrm{~S} ; \mathrm{ppm}\right)$, ammonia $\left(\mathrm{NH}_{3} ; \mathrm{ppm}\right)$ air velocity $(\mathrm{m} / \mathrm{s})$ and vapour density $\left(\mathrm{g} / \mathrm{m}^{3}\right)$ continuously during transport. The ambient temperature and relative humidity during transport were recorded continuously using TinyTalk dataloggers (Radionics, Dublin, Ireland). Environmental measurements, including gases $\left(\mathrm{NH}_{3}, \mathrm{H}_{2} \mathrm{~S}\right.$, $\left.\mathrm{CO}_{2}\right)$, relative humidity $(\mathrm{RH})$ and temperature $\left({ }^{\circ} \mathrm{C}\right)$, were recorded using QRae (Shawcity Ltd., UK), Testo 175 and Testo 445 portable multifunction probes (Testo UK, Ltd), respectively.

\section{Treatment groups}

Seventy two Charolais bulls (mean weight (s.d.) 367 (35) $\mathrm{kg}$ ) were randomly assigned by weight to one of six journey $(\mathrm{J})$ treatment times of 0 (no transport), 6 (J 6), 9 (J 9), 12 (J 12), 18 (J 18) and 24 (J 24) h transport ( $\mathrm{n}=$ 12 animals/treatment) at a stocking density of $1.02 \mathrm{~m}^{2} /$ bull. On the morning of the journey the bulls underwent social mixing, were moved from their home pens to a race with a chute to facilitate live weight recordings and blood sampling. The animals were blood sampled pretransport by jugular venipuncture to provide baseline physiological levels and again after the journey. Blood samples were collected by jugular venipuncture before $(-0.25 \mathrm{~h})$, immediately after $(0 \mathrm{~h})$ and at $1 \mathrm{~h}, 2 \mathrm{~h}, 4 \mathrm{~h}$, $6 \mathrm{~h}, 8 \mathrm{~h}, 12 \mathrm{~h}$ and $24 \mathrm{~h}$ relative to time $0 \mathrm{~h}$. Bulls were weighed using an animal weighing scale (Titan, Cattlemaster, Ireland) that was located at the exit area of the chute and rectal temperature was taken before, $-24 \mathrm{~h}$, $-0.25 \mathrm{~h}$, immediately after, and at 4,12 and 24 h relative to completion of the transport journey. The bulls were loaded onto the transporter and assigned to two pens on the transporter and remained in the same social groupings for the remainder of the study. The study animals were transported alone on the transporter. On completion of each of the transport journeys (J 6 - J 24) the bulls were returned for a $24 \mathrm{~h}$ rest period to novel pens $(n=2)$ with 6 animals/pen in the housing environment and had ad libitum access to water and grass silage supplemented with $2.0 \mathrm{~kg}$ barley/soybean concentrate.

Non-transported control animals (J 0) $(\mathrm{n}=12)$ were moved to two novel straw bedded pens ( 6 animals/pen) 
in the housing environment and animals had access to hay and water during the duration of the 'experimental' period coinciding with the maximum transport duration (24 h). Afterwards, control animals (J 0) had ad libitum access to grass silage supplemented with $2.0 \mathrm{~kg}$ barley/ soybean concentrate, water, and no hay for the $24 \mathrm{~h}$ period coinciding with the $24 \mathrm{~h}$ 'post-transport' period. Control animals (J 0) were blood sampled before assignment (at $-0.25 \mathrm{~h}$ ) to the novel pens, after $24 \mathrm{~h}$, and at 1 $\mathrm{h}, 2 \mathrm{~h}, 4 \mathrm{~h}, 6 \mathrm{~h}, 8 \mathrm{~h}, 12 \mathrm{~h}$ and $24 \mathrm{~h}$ relative to the completion of the $24 \mathrm{~h}$ experimental period. Control animals were weighed $-24 \mathrm{~h},-0.25 \mathrm{~h},+24 \mathrm{~h}$ and 4,12 , and $24 \mathrm{~h}$ after completion of the $24 \mathrm{~h}$ experimental period.

\section{Water intake}

Water consumption (litres) was recorded during transport and in the $24 \mathrm{~h}$ period post-transport. Flow metres were attached to the animal drinking containers and consumption was recorded on a pen basis in the housing environment and on the transporter.

\section{Rectal temperature}

The rectal body temperature was monitored before and after transport using a digital thermometer (Jorgen Kruuse A/S; Model VT-801BWC Lot No 0701).

\section{Assay procedures for physiological and haematological variables}

Heparinised blood samples were collected by jugular venipuncture and the plasma was separated by centrifugation at $1,600 \times \mathrm{g}$ at $8^{\circ} \mathrm{C}$ for 15 , and subsequently stored at $-20^{\circ} \mathrm{C}$ until assayed. Albumin, urea, globulin, total protein, $\beta$ hydroxybutyrate $(\beta \mathrm{HB})$, and creatine kinase (CK) were measured on an automated analyser (Olympus AU 400, Japan) using the reagents supplied by Olympus. Unclotted (EDTA-treated) whole-blood samples were collected by jugular venipuncture at the same time as the heparinised blood samples for haematological analysis. Red blood cell (RBC) number, white blood cell (WBC) number, differential WBC (percentage lymphocyte and neutrophil), haematocrit (HCT) percentage (\%), haemoglobin (Hgb) concentration, mean corpuscular volume (MCV), mean corpuscular haemoglobin concentration (MCHC) and platelet numbers were determined for unclotted (K $\mathrm{K}_{3}$-EDTA-treated) whole-blood samples with an automated cell counter (Celltac MEK-6108K; Nihon-Kohdon, Tokyo, Japan) within $1 \mathrm{~h}$ of blood sampling. Thin blood smears were also prepared on glass slides and stained using the haematology 3-step stain for differential WBC counts (Accralab, Biochemical Sciences; Fisher Scientific Company, Middletown, VA). Plasma haptoglobin concentration was determined as the haemoglobin binding capacity using an appropriate assay (TP801: Tridelta
Development Ltd., Greystones, Ireland), which was previously validated [32] and was quantified using a spACE automated analyser (Alfa Wassermann, Inc., West Caldwell, NJ, USA) [33]. Whole blood samples collected in sodium citrate tubes (Vacuette, Cruinn Diagnostics Limited, Ireland) were separated by centrifugation $(3000 \times \mathrm{g}$ at $8^{\circ} \mathrm{C}$ for 10 minutes) and the plasma frozen at $-20^{\circ}$ until assayed for fibrinogen using a commercial kit adapted for bovine plasma [51] (Randox Laboratories Ltd., Crumlin, Antrim, N. Ireland; catalogue No. GL2623) on an automated analyser (Hitachi 705, Boehringer Mannheim, Lewes, East Sussex, UK).

\section{Statistical analyses}

Physiological and haematological data, live weight data, water intake data and rectal temperature measurements were subjected to repeated measures using the PROC MIXED procedure of SAS (Version 9.1, SAS Institute, Cary, NC). Animal was the experimental unit and was specified as a repeated measures effect, and the dependence within animal was modelled using an unstructured covariance structure. The power of the statistical test was taken into consideration and was defined as the probability that the test will reject a false null hypothesis (i.e. that it will not make a Type II error). As power increases, the chances of a Type II error decrease. The probability of a Type II error is referred to as the false negative rate $(\beta)$, therefore power is equal to $1-\beta$ [52]. We calculated that with a group size of 12 bulls/treatment that the study would have $80 \%$ and greater power for the variables that we measured, meaning that if there was a difference of this given magnitude there would be an $80 \%$ chance of correctly detecting it as statistically significant. As " $n$ " the number of animals per treatment is the main determinant of power of test [52], the authors considered it crucial that adequate group sizes of animals were used, however, optimal sample size is ultimately a function of the underlying parameters in the study. In the present study, two pen replicates with 6 bulls/pen (total $\mathrm{n}=12$ ) were utilized for each transport journey. Using the measurements which were investigated in the present study, this sample size was deemed adequate without utilizing more animals than ethically necessary. Differences between means were tested using the PDIFF option in PROC MIXED in SAS. The PDIFF option calculates a separate probability value for each pair of means being compared. Means were considered significantly different at the $\mathrm{P}<0.05$ probability level [53].

\section{Acknowledgements}

The authors thank F. Collier, J. Larkin, A. Marley and M. Donlon, (Teagasc, Grange Beef Research Centre), for excellent technical help and assistance during the study. Many thanks are due to: the farm staff at Teagasc, Grange 
beef Research Centre, for care and management of the animals; $\mathrm{H}$. Mulligan, E. Mulligan, P. Reilly and G. Costelloe.

\section{Authors' contributions}

$\mathrm{BE}$ designed the study. BE, MM and DJP performed the experiments. BE analyzed the data and prepared the manuscript. All authors read and approved the final manuscript.

\section{Competing interests}

The authors declare that they have no competing interests.

Received: 19 May 2009 Accepted: 20 July 2010 Published: 20 July 2010

\section{References}

1. Kenny F J, Tarrant PV: The behaviour of young Friesian bulls during social re-grouping at an abbatoir. Influence of an overhead electrified wire grid. Appl Animal Beh Sci 1987, 18:233-246.

2. Kenny FJ, Tarrant PV: The physiological and behavioural responses of crossbred freisian steers to short haul transport by road. Livest Prod Sci 1987, 17:63-75

3. Earley B, O'Riordan EG: Effects on transporting bulls at different space allowances on physiological, haematological and immunological responses to a $12 \mathrm{~h}$ journey by road. Irish J of Agric and Food Res 2006, 45:39-50.

4. Earley B, AD Fisher, O'Riordan EG: Effects of pre-transport fasting on the physiological responses of young cattle to $8 \mathrm{~h}$ road transport. Irish $\mathrm{J}$ of Agric and Food Res 2006, 45:51-60.

5. Tarrant PV: Transportation of cattle by road. Appl Animal Beh Sci 1990, 28:153-170.

6. Tennessen T, Price MA, Berg RT: Comparative responses of bulls and steers to transportation. Can J Anim Sci 1984, 64:333-338.

7. Todd SE, Mellor DJ, Stafford KJ, Gregory NG, Bruce RA, Ward RN: Effects of food withdrawal and transport on 5 to 10 day old calves. Res Vet Sci 2000, 68(2):125-134.

8. Blecha F, Boyles SL, Riley JG: Shipping suppresses lymphocyte blastogenic responses in Angus and Brahman cross Angus feeder calves. J Anim Sci 1984, 59:576-583.

9. Blecha F, Baker PE: Effect of cortisol in vitro and in vivo on production of bovine interleukin 2. Am J Vet Res 1986, 47:841-845.

10. Murata $H$, Takahashi $H$, Matsumoto $H$ : The effect of road transportation on peripheral blood lymphocyte subpopulations, lymphocyte blastogenesis and neutrophil function in calves. Br Vet J 1987, 143:166-174.

11. Swanson J C, Morrow-Tesch J: Cattle transport: Historical, research, and future perspectives. J Anim Sci 2001, 79(E Supple):E102-E109.

12. Buckham Sporer KR, Burton $J$, Earley B, Crowe MA: Transportation stress in young bulls alters expression of neutrophil genes important for the regulation of apoptosis, tissue remodelling, margination, and antibacterial function. Vet Immunol Immunop 2007, 118:19-29.

13. Grandin T: Assessment of Stress during Handling and Transport. J Anim Sci 1997, 75:249-257.

14. Gupta S, Earley B, Crowe MA: Effect of $12 \mathrm{~h}$ road transportation on physiological, immunological, and haematological parameters in bulls housed at different space allowances. Vet J 2007, 173:605-616.

15. von Borell EH: The biology of stress and its application to livestock housing and transportation assessment. J Anim Sci 2001, 79(E Suppl): E260-E267.

16. Knowles TG: A review of the road transport of cattle. Vet Rec 1999, 144:197-201.

17. Tarrant PV, Kenny FJ, Harrington D, Murphy M: Long distance transportation of steers to slaughter: effect of stocking density on physiology, behaviour, and carcass quality. Livest Prod Sci 1992, 30:223-238.

18. SCAHAW: The welfare of animals during transport (details for horses, pigs, sheep and cattle). Report of the Scientific Committee on Animal Health and Animal Welfare (SCAHAW). European Commission: Brussels 2002, 130.

19. Warriss PD, Brown SN, Knowles TG, Kestin SC, Edwards JE, Dolan SK, Phillips AJ: Effects on cattle of transport by road for up to $15 \mathrm{~h}$. Vet Rec 1995, 136:319-323.
20. Arthington JD, Eicher SD, Kunkle WE, Martin FG: Effect of transportation and commingling on the acute-phase protein response, growth, and feed intake of newly weaned beef calves. J Anim Sci 2003, 81:1120-1125.

21. Galyean ML, Perino $\amalg$, Duff GC: Interaction of cattle health/immunity and nutrition. J Anim Sci 1999, 77:1120-1134.

22. Schaefer AL, Jones SDM, Tong AKW, Vincent BC: The effects of fasting and transport on beef cattle. 1. Acid-base-electrolyte balance and infra-red heat loss of beef cattle. Livest Prod Sci 1988, 20:15-24.

23. Atkinson PJ: Investigation of the effects of transport and lairage on hydration state and resting behaviour of calves for export. Vet Rec 1992, 130:413-416.

24. Crookshank HR, Elissalde MH, White RG, Clanton DC, Smalley HE: Effect of transportation and handling of calves upon blood serum composition. J Anim Sci 1979, 48:430-435.

25. Kent JE, Ewbank R: The effect of road transportation on the blood constituents and behaviour of calves. I. Six months old. Br Vet J 1983, 139:228-235.

26. Campbell NA, Reece JB, Mitchell LG: Biology Benjamin/Cummings, Menlo Park, CAMulligan E 1999, 907-910

27. Shaw FD, Tume RK: The assessment of pre-slaughter and slaughter treatments of livestock by measurement of plasma constituents - a review of recent work. Meat Sci 1992, 32:311-329.

28. Gagliardino JJ, Hernandez RE, Rebolledo OR: Chronobiological aspects of blood glucose regulation: a new scope for the study of diabetes mellitus. Chronobiologia 1984, 11:357-79.

29. Van Cauter E, Polonsky KS, Scheen AJ: Roles of circadian rhythmicity and sleep in human glucose regulation. Endocr Rev 1997, 18:716-38.

30. Tadich N, Gallo C, Aranis A: Blood concentration of some variables indicator of stress before and during exsanguination, in cattle. XII Congress of Veterinary Medicine. Chillán, Chile 2002.

31. Holmes JHG, Ashmorer CR, Robinson DW: Effect of stress on cattle with hereditary muscular hypertrophy. J Anim Sci 1973, 36:684-694.

32. Tarrant PV: Transportation of cattle by road. Appl Anim Behav Sci 1990 28:153-170.

33. Baumann H, Gauldie J: The acute phase response. Immunol Today 1994, 15:74-80.

34. Horadagoda N, Knox KMG, Gibbs HA, Reid WJ, Edwards SER, Eckersall PD: Acute phase proteins in cattle: discrimination between acute and chronic inflammation. Vet Rec 1999, 144:437-441.

35. Cheville NF: Introduction to Veterinary Pathology. Blackwell Publishing, Ames, lowa, USA, 32006.

36. Skinner JG, Brown RAL, Roberts L: Haptoglobin as an indicator of infection in sheep. Vet Rec 1994, 134:33-36.

37. Murata H, Miyamoto T: Bovine haptoglobin as a possible immunomodulator in the sera of transported calves. Br Vet J 1993, 149:277-283.

38. Tarrant PV, Kenny FJ, Harrington D, Murphy L: Long distance transportation of steers to slaughter: effect of stocking density on physiology, behaviour, and carcass quality. Livest Prod Sci Sci 1992, 30:223-238.

39. Leach TM: Physiology of the transport of cattle. "Transport of Animals Intended for Breeding Production and Slaughter Martinus Nijhoff, The NetherlandsMoss R 1981, 57-72.

40. Schalm OW: Ballieree Tindall and Cox Covent Garden London, UK 1961, 130-131.

41. Schalm OW: Manual of bovine haematology: Anemias/leukocytes/testing. Santa Barbara, Veterinary Practice Publishing 1984.

42. Jain NC: Schalm's Veterinary haematology. Philadelphia, Lea and Fibiger, 4 1986.

43. Kaneko JJ: Clinical biochemistry of domestic animals. Academic Press Inc. San Diego, 4 1989, 886-891.

44. Preisler MT, Weber PSD, Tempelman RJ, Erskine RJ, Hunt H, Burton JL: Glucocorticoid receptor down-regulation in neutrophils of periparturient cows. Am J Vet Res 2000, 61:14-19.

45. Burton UL, Madsen SA, Chang LC, Weber PSD, Buckham KR, Hickey MC, Earley B: Gene Expression Signatures in Neutrophils Exposed to Glucocorticoids: a New Paradigm to Explain "Neutrophil Dysfunction" in Parturient Dairy Cows. Vet Immunol Immunop 2005, 105(3-4):197-219.

46. Kent JE, Ewbank R: The effect of road transportation on the blood constituents and behaviour of calves II. One to three weeks old. Br Vet J 1986, 142:131-140. 
47. Kent JE, Ewbank R: The effect of road transportation on the blood constituents and behaviour of calves. III. Three months old. Br Vet J 1986, 142:326-335.

48. Ishizaki $H$, Hanafusa $Y$, Kariya $Y$ : Influence of truck-transportation on the function of bronchoalveolar lavage fluid cells in cattle. Vet Immunol Immunop 2004, 105:67-74.

49. Yagi Y, Shiono H, Chikayama Y, Ohnuma A, Nakamura I, Yayou K: Transport stress increases somatic cell counts in milk, and enhances the migration capacity of peripheral blood neutrophils of dairy cows. J Vet Med Sci 2004, 66:381-387.

50. Radostits OM, Blood DC, Gay CC: Veterinary medicine. A textbook of the diseases of cattle, sheep, pigs, goats and horses. Bailliere Tindall Ltd. London, UK, 8 1994, 967.

51. Earley B, Crowe MA: Effects of ketoprofen alone or in combination with local anesthesia during the castration of bull calves on plasma cortisol, immunological, and inflammatory responses. J Anim Sci 2002, 80:1044-1052.

52. Stroup WW: Power analysis based on spatial mixed effects analysis: a tool for comparing design and analysis strategies in the presence of spatial variability. J Agric Biol Environ Stat 2002, 7:491-511.

53. Snedecor GW, Cochran WG: "Statistical Methods". Iowa State University Press, Ames, IA USA, 8 1989, 503.

doi:10.1186/1746-6148-6-38

Cite this article as: Earley et al:: Effect of road transport for up to 24 hours followed by twenty-four hour recovery on live weight and physiological responses of bulls. BMC Veterinary Research 2010 6:38.

\section{Submit your next manuscript to BioMed Central and take full advantage of:}

- Convenient online submission

- Thorough peer review

- No space constraints or color figure charges

- Immediate publication on acceptance

- Inclusion in PubMed, CAS, Scopus and Google Scholar

- Research which is freely available for redistribution

Submit your manuscript at www.biomedcentral.com/submit

C) BioMed Central 